Z. klin. Chem. u. klin. Biochem.

8. Jg., S. 345-353, Juli 1970

\title{
Methoden zur Bestimmung von Östriol in der Schwangerschaft
}

\author{
Von B. RunNebaum und K. HolzmanN ${ }^{1}$ ) \\ Universitäts-Frauenklinik, Heidelberg (Direktor: Prof. Dr. J. Zander)
}

(Eingegangen am 17. März 1970)

Der Vergleich der verschiedenen Bestimmungsmethoden für Östriol führt zu folgenden Ergebnissen:

1. Es stehen für die Säurehydrolyse Methoden zur Verfügung, die bei verhältnismäßig kurzen Hydrolysezeiten gute Ausbeuten an Ostriol erlauben. Demgegenüber hat die enzymatische Hydrolyse den Nachteil ciner verbältnismäßig langen Inkubationsdauer.

2. Eine wesentliche Verbesserung der Ausbeute ist durch dic Gel-Filtration vor der Hydrolyse erreichbar. Sie führt cinmal zur Eliminierung von störenden Harnpigmenten und zur Entfernung von Glucose.

3. Für die Extraktion nach der Hydrolyse ist von wesentlicher Bedeutung, daß der verwandte Äther peroxidfrei ist. Optimale Ausbeuten an Ostriol werden bei der Extraktion nach Sättigung mit Ammoniumsulfat oder Natriumchlorid erreicht.

4. Die Empfindlichkeit der Methoden hängt weitgehend von spezifischen Reinigungsschritten wie Säulenchromatographie und Bildung von Östriol-Derivaten (z. B. 3-Methylöstriol) ab.

5. Für die quantitative Bestimmung von Ostriol bei Routinemethoden sind heute Farbreaktionen geeigneter als die Gaschromatographie.

6. Eine ausreichende Bestimmung der Zuverlässigkeitskriterien liegt bisher nur für einen Teil der Methoden vor. Bei diesen Methoden werden für die Richtigkeit Werte von $54-85 \%$ mit einer Standardabweichung von $3-10 \%$ und für die Genauigkeit Werte von $\pm 6-10 \%$ erreicht. Allerdings werden einzelne dieser Methoden bei der Bestimmung niedrigerer Östriolmengen in der Frühgravidität wesentlich ungenauer.

7. Von der 14. Schwangerschaftswoche an können heute etwa 6-20 Östriolbestimmungen innerhalb von höchstens 8 Stdn. zuverlässig vorgenommen werden. Doppelbestimmungen sind im Einzelfall ratsam.

\section{Methods for the determination of oestriol in preginancy}

Various methods for the determination of oestriol were compared:

1. The methods of acid hydrolysis give a good yield of oestriol in a relatively short hydrolysis time. By comparison, the enzymic hydrolysis has the disadvantage of a relatively long incubation period.

2. The yield can be considerably improved by gel filtration prior to hydrolysis. This removes interfering urinary pigments and glucose.

3. It is important that the extraction of the hydrolysate be performed with peroxide-free ether. Optimum yields of oestriol are obtained if the aqueous phase is saturated with ammonium sulphate or sodium chloride.

4. The sensitivity of the methods depends largely on specific purification steps, like column chromatography and the formation of oestriol derivates (e. g., 3-methyl-oestriol).

5. Colour reactions are more suitable nowadays than gas chromatography for the routine quantitative determination of oestriol.

6. A satisfactory determination of reliability criteria is only available for some of the methods; these show an accuracy factor of $54-85 \%$ with a standard deviation of $3-10 \%$ and values of $\pm 6-10 \%$ for the precision. Some of these methods are much less accurate in the determination of low concentrations of oestriol in early pregnancy.

7. From the 14th week of pregnancy upwards it is possible to perform 6-20 reliable oestriol determinations within $8 \mathrm{hrs}$. In individual cases duplicate determinations are advisable.

Die Forschungsergebnisse der letzten Jahre haben gezeigt, daß die Östriolaụsscheidung im Harn diagnostische Hinweise für den Zustand von Fetus und Placenta gibt. Es wurden zahlreiche Methoden für die quantitative Bestimmung von Östriol im Schwangerenharn entwickelt (Tab. 1). Zum Teil sind diese Methoden recht aufwendig und zeitraubend. Wir beschränken uns im folgenden auf eine kritische Gegenüberstellung der Methoden, welche für klinische Routinebestimmungen in Frage kommen.

\section{Methoden zur Hydrolyse, Extraktion und Reini- gung des Östriols}

Es ist das Ziel der Säurehydrolyse bei minimaler Destruktion eine möglichst hohe Ausbeute an Östriol zu erhalten. Seit den Arbeiten von StEvenson und MAR-

1) Vortrag, gehalten auf dem 15. Symposium der Deutschen Gesellschaft für Endokrinologie, 6.-8. März 1969 in Köln.
RIAN (1), Brown (2) und Bauld (3) hat die Hydrolyse mit $\left.15 \mathrm{Vol} .-\% \mathrm{HCl}^{2}\right)$ in zahlreichen Routinemethoden zur Bestimmung von Östriol Eingang gefunden, wobei die Lösung eine Stunde am Rückfluß erhitzt wird. Die Verluste an Östriol betragen unter diesen Bedingungen $10-20 \%$. BRown und BLAIR (4) fanden bei Erhöhung der HCl-Konzentration auf $20 \mathrm{Vol}-\mathrm{O} \%$ und Verkürzung der Hydrolysezeit auf 30 Min. gleich gute Resultate. Die Hydrolysezeit kann auf 15 Min. verkürzt werden durch Erhöhung der Temperatur auf $120^{\circ}$ (5). Die Verluste an Östriol waren unter den letztgenannten Bedingungen kleiner als $9 \%$. Ein weiterer Faktor, der die Wiederauffindung an Östriol bei der Hydrolyse beeinflußt, ist die Verdünnung des Harns. Die Zerstörung des Östriols bei der Säurehydrolyse kann nach vorheriger Reinigung des Harns durch Gel-Filtration, wie

2) Die Angabe Vol.-\% Säure wird hier gebraucht für die Volumenanteile konz. (37 proz. $\mathrm{w} / \mathrm{w}$ ) $\mathrm{HCl}$ bzw. konz. ( 96 proz. $\mathrm{w} / \mathrm{w}$ ) $\mathrm{H}_{2} \mathrm{SO}_{4}$. 
Tab. 1

Ostriolbestimmungsmethoden in der Schwangerschaft

\begin{tabular}{|c|c|c|c|c|c|c|}
\hline Autor & Hydrolyse & Extraktion & $\begin{array}{c}\text { Reinigung } \\
\text { Trrennung } \\
\text { der Óstrogene }\end{array}$ & $\begin{array}{c}\text { Trennung } \\
\text { des } \\
\text { Ostriols }\end{array}$ & $\begin{array}{l}\text { Derivat- } \\
\text { bildung }\end{array}$ & $\begin{array}{l}\text { Quantitative } \\
\text { Bestimmung }\end{array}$ \\
\hline $\begin{array}{l}\text { EBERLEIN und } \\
\text { Mitarbeiter (11) }\end{array}$ & $\begin{array}{l}500 \text { E Glucuronidase } \\
\text { pro } 20 \mathrm{ml} \text { Harn } \\
16-18 \mathrm{Stdn} ., 37^{\circ}\end{array}$ & $\begin{array}{l}\text { Ather } \\
2 \times 15 \mathrm{ml}\end{array}$ & $\begin{array}{l}\text { Verseifung mit } \\
\text { 1N NaOH } \\
\text { X̃therextraktion } \\
\text { Waschen mit } \mathrm{Na}_{2} \mathrm{CO}_{3}, \\
\mathrm{NaOH}+\mathrm{NaHCO}_{3}- \\
\text { Lösung }\end{array}$ & $\begin{array}{l}\text { Aluminium- } \\
\text { oxidsäule }\end{array}$ & - & $\begin{array}{l}\text { BATES und Mitar- } \\
\text { beiter }(50) \\
\text { Fluorometrie }\end{array}$ \\
\hline CAssmer (18) & $\begin{array}{l}\text { etwa } 200 \mathrm{ml} \text { Harn } \\
+15 \mathrm{ml} \text { conc. } \mathrm{HCl} \\
\text { I Std. kochen }\end{array}$ & $\begin{array}{l}\text { Ather } \\
1 \times 100 \mathrm{ml} \\
2 \times 50 \mathrm{ml}\end{array}$ & $\begin{array}{l}\text { Alkalisierung } \\
\text { Verteilung zw. Benzol/ } \\
\text { Hexan }(1: 1) \text { u. Wasser } \\
\text { Methylierung }\end{array}$ & $\begin{array}{l}\text { Aluminium- } \\
\text { oxidsäule }\end{array}$ & $\begin{array}{l}\text { 3-Methyl- } \\
\text { äther }\end{array}$ & $\begin{array}{l}\text { KOBER } \\
\text { Kolorimetrie }\end{array}$ \\
\hline $\begin{array}{l}\text { TAYLOR und } \\
\text { Mitarbeiter (40) }\end{array}$ & $\begin{array}{l}5 \mathrm{ml} \text { Harn auf } 100 \mathrm{ml} \\
\text { verdünnt }+15 \mathrm{ml} \\
\text { konz. HCl } 1 \mathrm{Std} . \\
\text { kochen }\end{array}$ & $\begin{array}{l}\text { Äther } \\
1 \times 50 \mathrm{ml} \\
2 \times 25 \mathrm{ml}\end{array}$ & $\begin{array}{l}\text { Alkalisierung Vertei- } \\
\text { lung zw. Benzol/ } \\
\text { Hexan }(1: 1) \text { u. Wasser } \\
\text { Methylierung }\end{array}$ & $\begin{array}{l}\text { Aluminium- } \\
\text { oxidsäule }\end{array}$ & $\begin{array}{l}\text { 3-Methyl- } \\
\text { äther }\end{array}$ & $\begin{array}{l}\text { KOBER } \\
\text { Kolorimetrie }\end{array}$ \\
\hline $\begin{array}{l}\text { Wotiz und } \\
\text { MARTIN }(31,41)\end{array}$ & $\begin{array}{l}100 \mathrm{ml} \text { Harn }+ \\
15 \text { Vol.\% konz. } \mathrm{HCl} \\
1 \text { Std. kochen }\end{array}$ & $\begin{array}{l}\text { Äther } \\
1 \times 100 \mathrm{ml} \\
2 \times 50 \mathrm{ml}\end{array}$ & Waschen mit $\mathrm{NaHCO}_{3}$ & - & Acetat & $\begin{array}{l}\text { Gaschromatographie } \\
4 \% \text { SE-30 Säule }\end{array}$ \\
\hline $\begin{array}{l}\text { PREEDY und } \\
\text { AITKEN (42) }\end{array}$ & $\begin{array}{l}85 \mathrm{ml} \text { Harn }+ \\
15 \mathrm{ml} \text { konz. HCl } \\
45 \mathrm{Min} \text {. kochen oder } \\
10-25 \mathrm{ml} \text { Schwange- } \\
\text { renplasma auf } 100 \mathrm{ml} \\
\text { verdünnt }+17,5 \mathrm{ml} \\
\text { konz. HCl, } 45 \text { Min. } \\
\text { kochen }\end{array}$ & $\begin{array}{l}\text { Äther } \\
4 \times 25 \mathrm{ml}\end{array}$ & $\begin{array}{l}\text { Waschen mit } \\
\text { NaHCO, Verteilung } \\
\text { zw. Toluol und } \\
\text { 1N NaOH } \\
\text { Ätherextraktion }\end{array}$ & Celitsäule & - & $\begin{array}{l}\text { BATES und Mit- } \\
\text { arbeiter }(50) \\
\text { Fluorometrie }\end{array}$ \\
\hline $\begin{array}{l}\text { KLOPPER und } \\
\text { WILSON (12) } \\
\text { KLOPPER und } \\
\text { STEPHENSON (43) }\end{array}$ & $\begin{array}{l}0,5-5 \mathrm{ml} \text { Harn auf } \\
20 \mathrm{ml} \text { verdünnt }+ \\
3 \mathrm{ml} \text { konz. HCl } \\
1 \text { Std. kochen }\end{array}$ & $\begin{array}{l}\text { Ather } \\
1 \times 20 \mathrm{ml}\end{array}$ & $\begin{array}{l}\text { Alkalisierung } \\
\text { Verseifung mit } \\
1 \mathrm{~N} \mathrm{NaOH} \\
\text { Âtherextraktion }\end{array}$ & $\begin{array}{l}\text { Aluminium- } \\
\text { oxidsäule }\end{array}$ & $\begin{array}{l}\text { 3-Methyl- } \\
\text { äther (wenn } \\
\text { mehr als } 5 \text { m } l \\
\text { Harn) }\end{array}$ & $\begin{array}{l}\text { KOBER } \\
\text { Kolorimetrie }\end{array}$ \\
\hline $\begin{array}{r}\text { STOA und } \\
\text { THORSEN (27) } \\
\vdots:\end{array}$ & $\begin{array}{l}20 \mathrm{ml} \text { Harn }+ \\
3 \mathrm{ml} \text { konz. HCl} \\
\text { l!Std. kochen }\end{array}$ & $\begin{array}{l}\text { Äther } \\
1 \times 20 \mathrm{ml} \\
2 \times 10 \mathrm{ml}\end{array}$ & $\begin{array}{l}\text { Alkalisierung } \\
\text { Verteilung zw. } \\
\text { 1N NaOH u. Benzol- } \\
\text { Petroläther }(1: 1)\end{array}$ & $\begin{array}{l}\text { Aluminium- } \\
\text { oxidsäule }\end{array}$ & - & $\begin{array}{l}\text { ITTRICH } \\
\text { Fluorometrie }\end{array}$ \\
\hline $\begin{array}{l}\text { TAYLOR und } \\
\text { Mitarbeiter (44) }\end{array}$ & $\begin{array}{l}5-20 \mathrm{ml} \text { Harn auf } \\
100 \mathrm{ml} \text { verdünnt }+ \\
15 \mathrm{ml} \text { konz. HCl } \\
2 \text { Stdn. kochen }\end{array}$ & $\begin{array}{l}\text { Äther } \\
1 \times 100 \mathrm{ml} \\
2 \times 50 \mathrm{ml}\end{array}$ & $\begin{array}{l}\text { Alkalisierung } \\
\text { Verseifung mit 1N } \\
\text { oder 2N NaOH } \\
\text { Ätherextraktion }\end{array}$ & $\begin{array}{l}\text { Aluminium- } \\
\text { oxidsäule }\end{array}$ & - & $\begin{array}{l}\text { KOBER } \\
\text { Kolorimetrie }\end{array}$ \\
\hline \multirow[t]{3}{*}{$\begin{array}{l}\text { BROWN und } \\
\text { COYLE (13) } \\
\text { (3 Methoden) }\end{array}$} & $\begin{array}{l}\text { bis } 50 \mathrm{ml} \text { Harn }+ \\
20 \text { Vol. } \% \text { konz. } \mathrm{HCl} \\
30 \mathrm{Min} \text {. kochen }\end{array}$ & $\begin{array}{l}\text { Äther } \\
1 \times 50 \mathrm{ml} \\
+7,5 \mathrm{~g} \mathrm{NaCl}\end{array}$ & $\begin{array}{l}\text { Alkalisierung } \\
\text { Verteilung } \mathrm{zw} \text {. Äther } \\
\text { u. 2N NaOH Methy- } \\
\text { lierung, Oxydation } \\
\text { mit } \mathrm{H}_{2} \mathrm{O}_{2}\end{array}$ & $\begin{array}{l}\text { Aluminium- } \\
\text { oxidsẫule }\end{array}$ & $\begin{array}{l}\text { 3-Methyl- } \\
\text { äther }\end{array}$ & $\begin{array}{l}\text { KOBER } \\
\text { Kolorimetrie }\end{array}$ \\
\hline & $\begin{array}{l}\text { bis } 50 \mathrm{ml} \text { Harn }+ \\
20 \text { Vol. \% konz. HCl } \\
30 \mathrm{Min} . \text { kochen }\end{array}$ & $\begin{array}{l}\text { Ather } \\
1 \times 50 \mathrm{ml} \\
+7,5 \mathrm{~g} \mathrm{NaCl}\end{array}$ & $\begin{array}{l}\text { Alkalisierung } \\
\text { Verteilung zw. Äther } \\
\text { u. } 2 \mathrm{~N} \mathrm{NaOH} \text { Methy- } \\
\text { lierung, Oxydation } \\
\text { mit } \mathrm{H}_{2} \mathrm{O}_{2}\end{array}$ & - & $\begin{array}{l}\text { 3-Methyl- } \\
\text { äther }\end{array}$ & $\begin{array}{l}\text { KOBER } \\
\text { Kolorimetrie }\end{array}$ \\
\hline & $\begin{array}{l}\text { bis } 50 \mathrm{ml} \text { Harn }+ \\
20 \text { Vol. \% konz. HCl } \\
30 \text { Min. kochen }\end{array}$ & $\begin{array}{l}\text { Äther } \\
1 \times 50 \mathrm{ml} \\
+7,5 \mathrm{~g} \mathrm{NaCl}\end{array}$ & $\begin{array}{l}\text { Nur Verseifung } \\
\text { mit in NaOH }\end{array}$ & - & - & $\begin{array}{l}\text { KOBER } \\
\text { Kolorimetrie }\end{array}$ \\
\hline Beling (16) & $\begin{array}{l}\text { 2-6 ml Sephadex } \\
\text { G25 gereinigter Harn, } \\
200 \mathrm{FU} \beta \text {-Glucuronidase } \\
\text { pro ml } 16 \text { Stdn., } 37^{\circ}\end{array}$ & $\begin{array}{l}\text { Äther } \\
2 \times \text { gleiches } \\
\text { Volumen }\end{array}$ & $\begin{array}{l}\text { Sephadex G25 } \\
\text { Verteilung zw. Benzol/ } \\
\text { Hexan } 1: 1 \text { u. Wasser } \\
\text { Methylierung }\end{array}$ & - & $\begin{array}{l}\text { 3-Methyl- } \\
\text { äther }\end{array}$ & $\begin{array}{l}\text { KOBER (NOCKE (25) } \\
\text { Kolorimetrie }\end{array}$ \\
\hline $\begin{array}{l}\text { GREEN und } \\
\text { TOUCHSTONE (14) }\end{array}$ & $\begin{array}{l}5 \mathrm{ml} \text { Harn auf } 20 \mathrm{ml} \\
\text { verdünnt }+3 \mathrm{ml} \\
\text { konz. HCl } 1 \mathrm{Std} \text {. } \\
\text { kochen }\end{array}$ & $\begin{array}{l}\text { Äther } \\
1 \times 22-25 \\
\mathrm{ml}\end{array}$ & $\begin{array}{l}\text { wie EBERLEIN und } \\
\text { Mitarbeiter (11) }\end{array}$ & $\begin{array}{l}\text { Aluminium- } \\
\text { oxidsãule }\end{array}$ & $一$ & $\begin{array}{l}\text { BACHMANN-Reak- } \\
\text { tion } \\
\text { Kolorimetrie }\end{array}$ \\
\hline $\begin{array}{l}\text { TOUCHSTONE und } \\
\text { Mitarbeiter (45) }\end{array}$ & $\begin{array}{l}10 \mathrm{ml} \text { Schwangeren- } \\
\text { plasma }+15 \text { Vol. } \% \\
\text { konz. HCl } \\
1 \text { Std. kochen }\end{array}$ & $\begin{array}{l}\text { Äther } \\
3 \times \text { gleiches } \\
\text { Volumen }\end{array}$ & $\begin{array}{l}\text { Waschen mit } \\
\text { alkalischer Lösung } \\
\text { u. Wasser }\end{array}$ & $\begin{array}{l}\text { Aluminium- } \\
\text { oxidsäule }\end{array}$ & - & $\begin{array}{l}\text { BACHMANN-Reak- } \\
\text { tion } \\
\text { Kolorimetrie }\end{array}$ \\
\hline \multirow[t]{2}{*}{$\begin{array}{l}\text { FRANDSEN und } \\
\text { STAKEMANN } \\
(37,46) \\
(2 \text { Methoden) }\end{array}$} & $\begin{array}{l}\text { etwa } 5 \mathrm{ml} \text { Harn auf } \\
10 \mathrm{ml} \text { verdünnt }+ \\
3 \text { Vol. } \% \mathrm{H}_{2} \mathrm{SO}_{4} \\
1 \text { Std. } 120^{\circ}\end{array}$ & $\begin{array}{l}\text { Ather } \\
1 \times 25 \mathrm{ml}\end{array}$ & $\begin{array}{l}\text { Alkalisierung } \\
\text { Verteilung } \mathrm{zw}_{\text {. }} \\
\text { 2N NaOH }+ \text { Ather }\end{array}$ & $\begin{array}{l}\text { Verteilung } \\
\text { zwischen } \\
\text { Lösungs- } \\
\text { mitteln }\end{array}$ & - & $\begin{array}{l}\text { KOBER } \\
\text { Kolorimetrie }\end{array}$ \\
\hline & $\begin{array}{l}\text { etwa } 20 \mathrm{ml} \text { Harn auf } \\
40 \mathrm{ml} \text { verdünnt }+ \\
5 \mathrm{~g}(\mathrm{NH},)_{2} \mathrm{SO}_{4} \\
1 \text { Std. } 120^{\circ}\end{array}$ & $\begin{array}{l}\text { Ather } \\
1 \times 40 \mathrm{ml}\end{array}$ & $\begin{array}{l}\text { Alkalisierung } \\
\text { Verteilung } \mathrm{zw}_{\text {. }} \\
\text { 2N NaOH }+ \text { Aher }\end{array}$ & $\begin{array}{l}\text { Aluminium- } \\
\text { oxidsäule }\end{array}$ & - & $\begin{array}{l}\text { KOBER } \\
\text { Kolorimetrie }\end{array}$ \\
\hline TOUCHSTONE (33) & $\begin{array}{l}5 \mathrm{ml} \text { Harn auf } \\
15 \mathrm{ml} \text { verdünnt }+ \\
15 \mathrm{Vol} \% \mathrm{HCl} \\
15 \mathrm{Min} . \text { Kochen }\end{array}$ & $\begin{array}{l}\text { Äther } \\
2 \times \text { gleiches } \\
\text { Volumen }\end{array}$ & $\begin{array}{l}\text { Verteilung zw. } \\
\text { 1N NaOH u. Äther } \\
\text { Waschen mit NaHCO, } \\
\text { u. Wasser }\end{array}$ & 一 & - & Gaschromatographie \\
\hline YOUSEM (32) & $\begin{array}{l}200 \mathrm{ml} \mathrm{Harn}+30 \mathrm{ml} \\
\text { konz. HCl } \\
1 \text { Std. kochen }\end{array}$ & $\begin{array}{l}\text { Äther } \\
1 \times 200 \mathrm{ml} \\
2 \times 100 \mathrm{ml}\end{array}$ & $\begin{array}{l}\text { Waschen mit NaHCO, } \\
\text { u. Wasser. Verteilung } \\
\text { zw. Toluol u. IN NaOH }\end{array}$ & - & 一 & $\begin{array}{l}\text { Gaschromatographie } \\
2 \% \text { QF-1 Säule }\end{array}$ \\
\hline $\begin{array}{l}\text { DALE und } \\
\text { Mitarbeiter (29) }\end{array}$ & $\begin{array}{l}5 \mathrm{ml} \text { Harn auf } \\
20 \mathrm{ml} \text { verdünnt }+ \\
3 \mathrm{ml} \text { konz. HCl } \\
1 \text { Std. kochen }\end{array}$ & $\begin{array}{l}\text { Äther } \\
2 \times 22 \mathrm{ml}\end{array}$ & $\begin{array}{l}\text { Waschen mit } \\
\text { alkalischen Lösungen }\end{array}$ & $\begin{array}{l}\text { Aluminium- } \\
\text { oxidsäule }\end{array}$ & 一 & $\begin{array}{l}\text { BACHMAN-Reak- } \\
\text { tion } \\
\text { Kolorimetrie }\end{array}$ \\
\hline $\begin{array}{l}\text { ADLERCREUTZ und } \\
\text { LUUKKAINEN (47) }\end{array}$ & $\begin{array}{l}10 \mathrm{ml} \text { Sephadex-G25 } \\
\text { gereinigter Harn } \\
600 \mathrm{FU}-\beta \mathrm{Glucuronidase} \\
+6000 \mathrm{E} \\
\text { Phenolsulfatase } \\
16 \text { Stdn. } 37^{\circ} \\
\text { oder Hydrolyse nach } \\
\text { BRowN (2) }\end{array}$ & $\begin{array}{l}\text { Ather } \\
3 \times \text { gleiches } \\
\text { Volumen }\end{array}$ & $\begin{array}{l}\text { Sephadex-G25 } \\
\text { Verteilung zw. } \\
\text { 1N NaOH u. Äther } \\
\text { Methylierung }\end{array}$ & 一 & $\begin{array}{l}16 \alpha, 17 \beta- \\
\text { dimethyl- } \\
\text { silyläther } \\
\text { des Ostriol- } \\
\text { 3-methyl- } \\
\text { äthers }\end{array}$ & $\begin{array}{l}\text { Gaschromatographie } \\
\text { Z, NGS oder SE-30 } \\
\text { Säulen } \\
\text { KOBER } \\
\text { (NOCKE (25) }\end{array}$ \\
\hline $\begin{array}{l}\text { NACHTIGALL und } \\
\text { Mitarbeiter (38) }\end{array}$ & $\begin{array}{l}1 \mathrm{ml} \text { Plasma }+0,2 \mathrm{ml} \\
\left.\text { Ostriol- }{ }^{3} \mathrm{H}\right]-\mathrm{Konjugate} \\
+2,8 \mathrm{ml} \text { Wasser }+ \\
15 \mathrm{Vol} \% \mathrm{konz} . \mathrm{HCl} \\
40 \mathrm{Min} .100-110^{\circ}\end{array}$ & $\begin{array}{l}\text { Äther } \\
4 \times 5 \mathrm{ml}\end{array}$ & $\begin{array}{l}\text { Verteilung zw. } \\
\text { Benzol/Hexan (1:1) } \\
\text { u. Wasser } \\
\text { Ätherextraktion }\end{array}$ & $\begin{array}{l}\text { Verteilung } \\
\text { zwischen } \\
\text { Lösungs- } \\
\text { mitteln }\end{array}$ & 一 & $\begin{array}{l}\text { KOBER } \\
\text { ITTRICH } \\
\text { Fluorometrie }\end{array}$ \\
\hline
\end{tabular}


Tab. 1 (Fortsetzung)

\begin{tabular}{|c|c|c|c|c|c|c|}
\hline Autor & Hydrolyse & Extraktion & $\begin{array}{l}\text { Reinigung } \\
\text { Trennung } \\
\text { der Ôstrogene }\end{array}$ & $\begin{array}{l}\text { Trennung } \\
\text { des } \\
\text { Ostriols }\end{array}$ & $\begin{array}{l}\text { Derivat- } \\
\text { bildung }\end{array}$ & $\begin{array}{l}\text { Quantitative } \\
\text { Bestimmung }\end{array}$ \\
\hline $\begin{array}{l}\text { SCHINDLER und } \\
\text { HERRMANN (22) }\end{array}$ & $\begin{array}{l}50 \mathrm{ml} \text { Harn + Ostriol- } \\
{\left[6,7-^{2} \mathrm{H}\right]} \\
+15 \text { Vol. } \% \text { konz. } \mathrm{HCl} \\
1 \text { Std. kochen }\end{array}$ & $\begin{array}{l}\text { Ather } \\
3 \times 50 \mathrm{ml}\end{array}$ & $\begin{array}{l}\text { Waschen mit NaHCO, } \\
\text { Verteilung zw. } \\
\text { 2N NaOH } \\
\text { Á. Ather } \\
\text { Athextraktion }\end{array}$ & - & $\begin{array}{l}\text { Trimethyl- } \\
\text { silyläther }\end{array}$ & $\begin{array}{l}\text { Gaschromatographie } \\
2 \% \times E-60 \text { Säule }\end{array}$ \\
\hline $\begin{array}{l}\text { BENGTSSON und } \\
\text { FORSGREN (20) }\end{array}$ & $\begin{array}{l}200 \mathrm{ml} \text { Harn }+30 \mathrm{ml} \\
\text { konz. HCl } \\
30 \mathrm{Min} . \text { kochen }\end{array}$ & $\begin{array}{l}\text { Ather } \\
1 \times 100 \mathrm{ml} \\
2 \times 50 \mathrm{ml}\end{array}$ & $\begin{array}{l}\text { Waschen mit NaHCO, } \\
\text { Vertellung } 2 \text { W }_{3} \\
\text { IN NaOH u. Ather } \\
\text { Átherextraktion }\end{array}$ & - & Acetat & $\begin{array}{l}\text { Gaschromatographie } \\
\mathbf{3 \%} \text { SE-30 Säule }\end{array}$ \\
\hline HAUSKNECHT (23) & $\begin{array}{l}100 \mathrm{ml} \text { Harn auf } \\
200 \mathrm{ml} \text { verdünnt }+ \\
\text { Ostriol-[7-3 } \mathrm{H}]+30 \mathrm{ml} \\
\text { konz. HCl } \\
45 \mathrm{Min} \text {. kochen }\end{array}$ & $\begin{array}{l}\text { Ather } \\
\text { mehrmals }\end{array}$ & $\begin{array}{l}\text { Verteilung zw } \\
\text { 2N NaOH u. Ẍther } \\
\text { Atherextraktion }\end{array}$ & $\begin{array}{l}\text { Dünnschicht- } \\
\text { chromato- } \\
\text { graphie }\end{array}$ & $\begin{array}{l}\text { Trimethyl- } \\
\text { silyläther } \\
\text { (nur für Gas- } \\
\text { chromato- } \\
\text { graphie) }\end{array}$ & $\begin{array}{l}\text { KOBER- } \\
\text { ITTRICH } \\
\text { Kolorimetrie } \\
\text { Gaschromatographie }\end{array}$ \\
\hline $\begin{array}{l}\text { NILSSON und } \\
\text { BENGTSSON (21) }\end{array}$ & $\begin{array}{l}50.000 \mathrm{E} \beta \text {-Glucuro- } \\
\text { nidase }+50.000 \mathrm{E} \\
\text { Sulfatase } \\
90 \text { Stdn. } 37^{\circ}\end{array}$ & $\begin{array}{l}\text { Xtherl } \\
\text { Áthanol } \\
4: 1 / \mathrm{v}: \mathrm{v} \\
3 \times 100 \mathrm{ml}\end{array}$ & $\begin{array}{l}\text { Waschen mit } \mathrm{NaHCO}_{3} \\
\text { Verteilungen zw. } \\
\text { 2N NaOH u. Xther }+ \\
\text { 1N NaOH u. Toluol } \\
\text { Átherextraktion }\end{array}$ & $\begin{array}{l}\text { Verteilung } \\
\text { zwischen } \\
\text { Lösungs- } \\
\text { mitteln }\end{array}$ & Acetat & $\begin{array}{l}\text { Gaschromatographie } \\
2,4 \% \text { XE-60 } \\
\text { Säule }\end{array}$ \\
\hline
\end{tabular}

Tab. 2

Säurehydrolyse für Ostriolbestimmungen in der Schwangerschaft

\begin{tabular}{|c|c|c|c|c|c|c|}
\hline Harn & $\mathrm{ml}$ & Säurezusatz & Std. & $\begin{array}{l}\text { Inkubation } \\
\text { Temperatur }\end{array}$ & - Verluste & Autor \\
\hline $\begin{array}{l}\text { Nativharn } \\
\text { Nativharn } \\
\text { Gel-Filtrat } \\
\text { auf } 6 \mathrm{ml} \\
\text { verdünnt }\end{array}$ & $\begin{array}{r}200 \\
50 \\
10 \\
0,5-4\end{array}$ & $\begin{array}{l}30 \mathrm{ml} \mathrm{HCl} \\
20 \mathrm{Vol} \% \% \mathrm{HCl} \\
15 \mathrm{Vol} \% \% \mathrm{HCl} \\
15 \text { Vol. } \% \mathrm{HCl}\end{array}$ & $\begin{array}{l}1 \\
2 / 2 \\
1 \\
2 / 4\end{array}$ & $\begin{array}{l}100^{\circ} \\
100^{\circ} \\
100^{\circ} \\
120^{\circ}\end{array}$ & $\begin{array}{c}10-20 \\
10-20 \\
0-5 \\
9\end{array}$ & $\begin{array}{l}\text { BROWN (2) } \\
\text { BROWN Und COYLE (13) } \\
\text { ADLERCREUTZ und LUUKKAINEN (7) } \\
\text { BROWN Und Mitarbeiter (5) }\end{array}$ \\
\hline $\begin{array}{l}\text { auf } 20 \mathrm{ml} \\
\text { verdünnt }\end{array}$ & 5 & 3 Vol. $\% \mathrm{H}_{3} \mathrm{SO}$ & 1 & $120^{\circ}$ & 5 & FRANDSEN (8) \\
\hline
\end{tabular}

Beling (6) und Adlercreutz und LuUkrainen (7) zeigten, unter $5 \%$ gehalten werden. FrANDSEN (8) hydrolysierte mit 3 Vol.- $\% \mathrm{H}_{2} \mathrm{SO}_{4}$ eine Stunde bei $120^{\circ}$ und hat durchschnittlich $5 \%$ Verluste an Östriol. Einige Hydrolyseverfahren sind in Tabelle 2 zusammengestellt.

Bei unverdünntem Harn (Abb. 1) wird mit 2-3 Vol.-\% Schwefelsäure ein Hydrolyseoptimum für Östriol erreicht. Bei höherer Säurekonzentration kommt es unter den gleichen Bedingungen zu erheblichen Verlusten. Ist jedoch der Harn $1: 5$ verdünnt, dann sind selbst bei Verwendung von 6 Vol.-\% Schwefelsäure die Verluste noch verhältnismäßig gering. $\mathrm{Daß}$ die Pigmentkonzentration im Harn bei der Hydrolyse die Ausbeute an

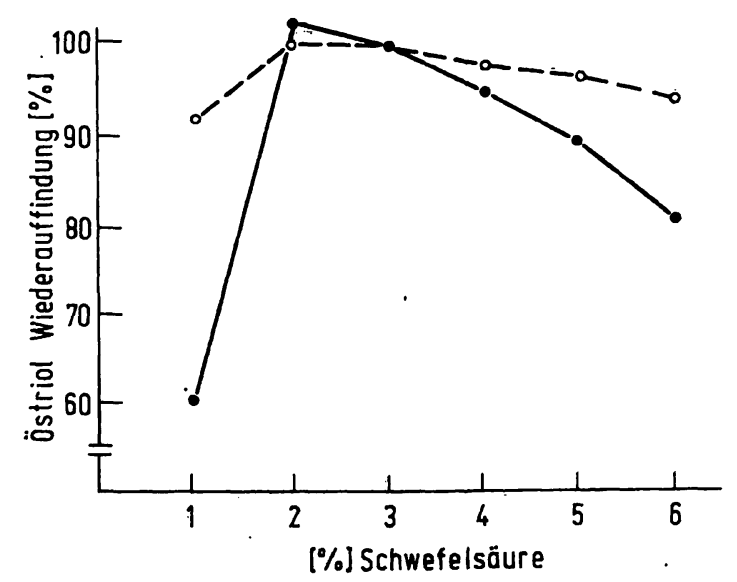

Abb. 1

Wiederauffindung von Östriol nach Hydrolyse mit unterschiedlichen Schwefelsäurekonzentrationen bei $120^{\circ}$ und 1 Std. Erhitzen.. Es wurden unverdünnter (•-•) und $1: 5$ verdünnter $(0-\cdots)$ Harn verwandt (nach FRANDSEN, (8))
Östriol beeinflussen kann, ist bekannt. So fand HoBkIRK (9) im Harn einer Schwangeren, der 1:2,5 verdünnt war, 15,4 mg Östriol pro 24 Stundenharn, während in demselben Harn, der 1:24fach verdünnt war, $22,1 \mathrm{mg}$ pro 24 Stundenharn gefunden wurde.

Ferner kann Glucose bei der Säurehydrolyse die Wiederauffindung von Östriol erheblich stören. Um diese Fehlermöglichkeit zu umgehen, bieten sich verschiedene Verfahren an. Einmal die Verdünnung des Harns auf $1: 10(4,9)$, die Gel-Filtration zur Entfernung der Glucose (6) und die Sättigung des glucosehaltigen Harns mit Ammoniumsulfat, wobei konjugierte Östrogene mit Essigester: Äthanol (23: 7, v/v) extrahiert werden. Die letzte Methode hat FRANDSEN (10) angewandt.

Die Säurehydrolyse kann bei ausreichender Ausbeute an Östriol mit verhältnismäßig großen Harnmengen im Vergleich zur enzymatischen Hydrolyse schnell und billig durchgeführt werden. Sie eignet sich daher besonders für Routinemethoden.

Bei der enzymatischen Hydrolyse schwanken die Östriolausbeuten gelegentlich außerordentlich stark. Das liegt einmal an der unterschiedlichen Aktivität der Enzympräparate, zum anderen an Enzyminhibitoren, die im Harn in wechselndem Umfange entstehen können. Die Entstehung solcher Enzyminhibitoren läßt sich durch eine vorherige Gel-Filtration des Harns weitgehend ausschalten. Beling (6) sowie AdLERCReutz und LUUKKAINEN (7) fanden nach Gel-Filtration und anschließender enzymatischer Hydrolyse eine mittlere Wiederauffindung an Östriol von 95\%. Die kürzesten Zeiten, die für enzymatische Hydrolyse in Östriolbestimmungsmethoden genannt werden, betragen $16 \operatorname{Stdn} .(6,7$, 11). 


\section{Methoden zur Extraktion}

Die Extraktion des hydrolysierten Harns erfolgt in den meisten Östriolbestimmungsmethoden mit Diäthyläther. Genauere Daten für die Ausbeute an Östriol bei der Ätherextraktion liegen nur vereinzelt vor. Bemessen an der Wiederfindung der Gesamtmethode scheint eine einmalige Extraktion mit gleichem Volumen Äther ausreichend zu sein, wenn dem Harn vor der Hydrolyse Natriumchlorid oder Ammoniumsulfat zugesetzt wird $(5,8,12$, 13, 14). Östriol läßt sich nahezu quantitativ extrahieren, wenn der hydrolysierte Harn mindestens eine 12proz. Ammoniumsulfatsättigung aufweist (Abb. 2).

Ferner spielt die für Extraktion von Östriol der Peroxidgehalt des Äthers eine wesentliche Rolle. So hat FRANDSEN (8) aus Peroxid-Äther, dem $10 \mu \mathrm{g}$ Östriol zugesetzt waren, nur 5,6 $\mu \mathrm{g}$ Östriol wiedergefunden.

Zur Reinigung des Äthers werden Eisensulfat-, Silbernitrat-Lösungen sowie Kalilauge verwendet $(8,15,16)$. Der Äther sollte wöchentlich $2 \mathrm{mal}$ präpariert und bei $4^{\circ}$ aufbewahrt werden.

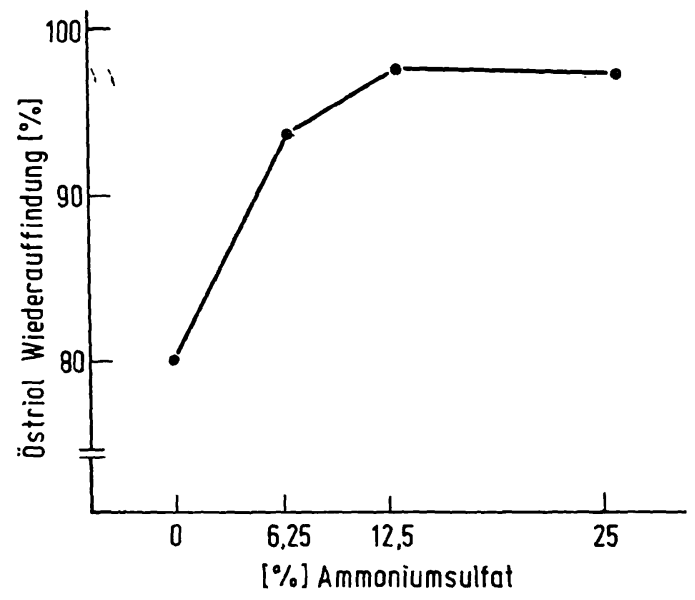

Abb. 2

Wiederauffindung von Óstriol bei der Extraktion des hydrolysierten Harns mit Äther nach unterschiedlichem Zusatz von Ammoniumsulfat (nach FRANDSEN, (8))

\section{Reinigung und Trennung des Östriols}

Die Trennung der phenolischen Östrogene von den neutralen Steroiden erfolgt gewöhnlich durch Verteilung zwischen organischen Lösungsmitteln und wäßr. $\mathrm{NaOH}-L o ̈ s u n g e n ~(3,17)$. In den meisten Östriolmethoden erfolgt zusätzlich entweder eine Trennung des Östriols durch Säulenchromatógraphie an Aluminiumoxid oder eine Derivatbildung des Östriols (Abb. 3). In einigen Methoden mit kolorimetrischer Bestimmung des Östriols wird die Bildung des 3-Methyläthers nach BRown (2) angewandt $(18,13,6)$. Für die gaschromatographische Bestimmung des Östriols haben sich Acetate $(19,20,21)$ und Dimethylsilyl-Äther als brauchbar erwiesen $(7,22)$. Besondere Vorteile für die Reinigung und Trennung des Östriols bringt die von Beling (6) angegebene Gel-Filtration an Sephadex G 25, da neben der Fraktionierung der Östriolkonjugate vor der Hydrolyse schon Harnpigmente und beispielsweise Glucose entfernt werden.

Die Dünnschichtchromatographie wurde für die Isolierung des Östriols aus Schwangerenharn von HAusKNECHT (23) angewandt. Im übrigen haben aber Dünnschicht- und Papierchromatographie für routinemäßige Östriolbestimmungen bisher keine Anwendung gefunden.

\section{Farbreaktionen zur quantitativen Bestimmung von} Östriol

KOBER (24) fand, daß phenolische Östrogene durch Erhitzen mit Schwefelsäure einen gelben Farbkomplex bilden, der nach Verminderung der Schwefelsäurekonzentration in rot übergeht. Zur optimalen Bildung des ersten Farbkomplexes wird für Östriol 76\% Schwefelsäure bei Anwesenheit von Hydrochinon verwandt, während zur Entstehung des zweiten Farbkomplexes nach BRown (2) $55 \%$ Schwefelsäure erforderlich ist. NockE (25) hat die Bedingungen für die Bildung der

\begin{tabular}{|c|c|c|c|c|c|c|c|c|c|c|}
\hline $\begin{array}{l}\text { Reinigung des } \\
\text { Östriols und } \\
\text { quantitative } \\
\text { Bestimmung }\end{array}$ & $\left|\begin{array}{c}\text { Taylor } \\
\text { und Mit- } \\
\text { arbeiter } \\
(40)\end{array}\right|$ & (6) & $\begin{array}{l}\text { Greene } \\
\text { und } \\
\text { Touch- } \\
\text { stone } \\
(14)\end{array}$ & $\left|\begin{array}{c}\text { Frandsen } \\
\text { und } \\
\text { Stake- } \\
\text { mann } \\
(37,46)\end{array}\right|$ & $\begin{array}{l}\text { schindler } \\
\text { und } \\
\text { Herr- } \\
\text { mann } \\
\text { (22) }\end{array}$ & $\begin{array}{c}\text { Klopper } \\
\text { und } \\
\text { Stephen- } \\
\text { son } \\
(43)\end{array}$ & $\begin{array}{c}\text { Haus- } \\
\text { knecht } \\
(23)\end{array}$ & $\begin{array}{l}\text { Brown } \\
\text { und } \\
\text { Coyle } \\
(13)\end{array}$ & $\left|\begin{array}{c}\text { Brown } \\
\text { und Mit- } \\
\text { arbeiter } \\
(5)\end{array}\right|$ & . \\
\hline Sephadex & & 9 & & & & & & & & \\
\hline $\begin{array}{l}\text { Verteilung organ. } \\
\text { Lösungsmittel: } \mathrm{NaOH} \\
\text { Verteilung organ. } \\
\text { Lösungsmittel: } \mathrm{H}_{2} \mathrm{O}\end{array}$ & 9 & & 9 & & $i$ & $?$ & 9 & $i$ & 9 & Abb. 3 \\
\hline $\begin{array}{l}\text { Methylierung } \\
\mathrm{Al}_{2} \mathrm{O}_{3} \text {-Säule } \\
\text { Dünnschicht- } \\
\text { Chromatographie }\end{array}$ & $\phi$ & & & i & & & & & & $\begin{array}{l}\text { Darstellung der wesentlichen } \\
\text { Schritte zur Reinigung und } \\
\text { Trennung von Ostriol sowie } \\
\text { der Meßpirinzipien einiger } \\
\text { Ostriolbestimmungsmethoden }\end{array}$ \\
\hline $\begin{array}{l}\text { Trimethyl-Silyl- } \\
\text { Derivat }\end{array}$ & & & & & & & & & & $\begin{array}{l}\bullet=\text { obligater Schritt } \\
\stackrel{0}{=} \text { fakultativer Schritt }\end{array}$ \\
\hline Kober & & & & d & & I & & d & $\phi$ & \\
\hline Ittrich & & & & & & & & & d & \\
\hline Bachmann & & & $\boldsymbol{b}$ & & & & & & & \\
\hline $\begin{array}{l}\text { Gas- } \\
\text { chromatographie }\end{array}$ & & & & & & & & & & \\
\hline
\end{tabular}




\section{Standardmathoden}

itr
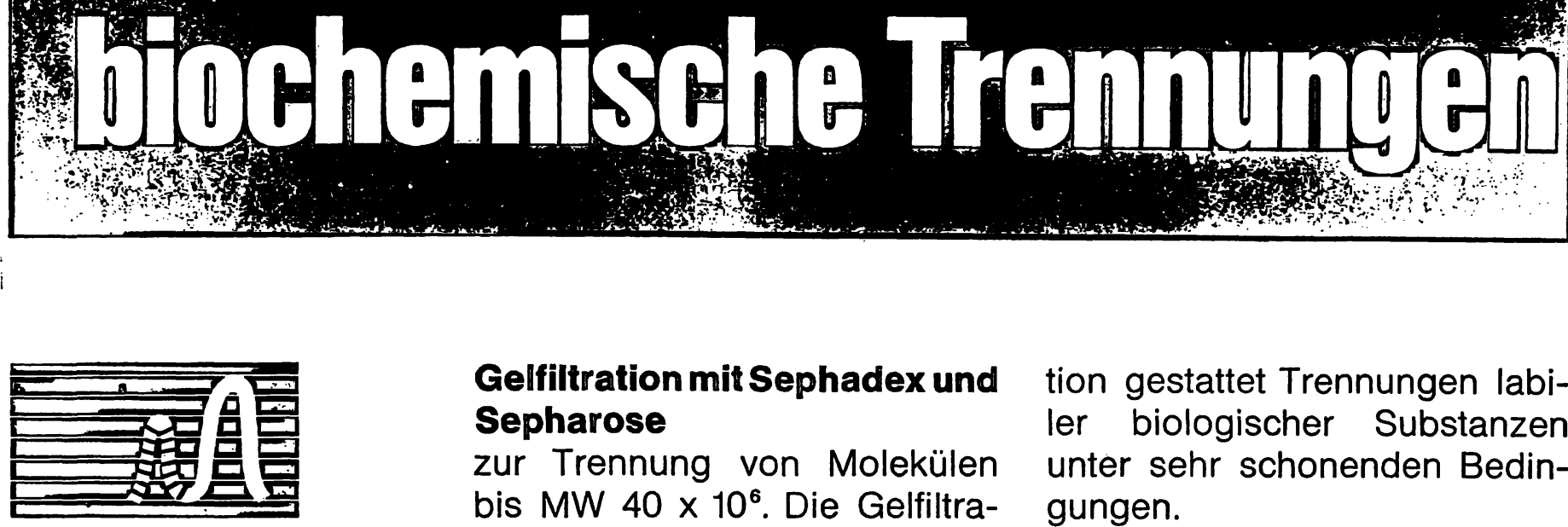

Gelfiltration mit Sephadex und Sepharose

zur Trennung von Molekülen bis MW $40 \times 10^{6}$. Die Gelfiltra- tion gestattet Trennungen labiler biologischer Substanzen unter sehr schonenden Bedingungen.
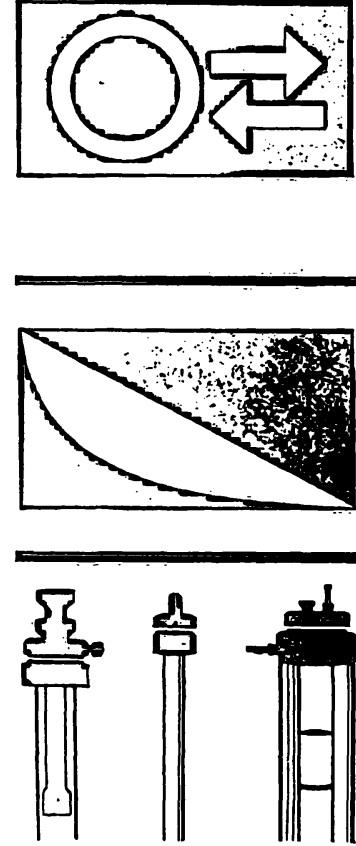

lonenaustauscher-Chromatographie mit Sephadex-Ionenaustauschern,

die die Vorzüge von CelluloseundKunstharzaustauschern ver- einigen. Charakteristisch sind hohe Kapazität, niedrige unspezifische Adsorption und ausgezeichnete Reproduzierbarkeit.
Verteilung in wäßrigen Phasen-Systemen mit Dextran und Dextran-Derivaten zur Fraktionierung sehr hochmolekularer Stoffe wie Viren, Nukleinsäuren und Zellpartikeln unter sehr milden Bedingungen.

graphie. entwickelt haben, ermöglichen reproduzierbare Trennresultate. Sie stehen Ihnen in großer Auswahl mit diversem Zubehör zur Verfügung.

\section{Literaturdienst}

Als Hilfe für Wissenschaftler geben wir einen umfassenden Literaturdienst heraus. Eine $\frac{2}{2}$ के jährlich erscheinende Referenzliste enthält etwa 1000 neue
Literaturstellen. Bitte schreiben Sie uns, wenn wir Sie in unseren Verteiler aufnehmen sollen. Fordern Sie auch Broschüren über unsere Separationsprodukte und das Literaturverzeichnis an. 


\section{Sie können in}

noch kürzerer Zeit für

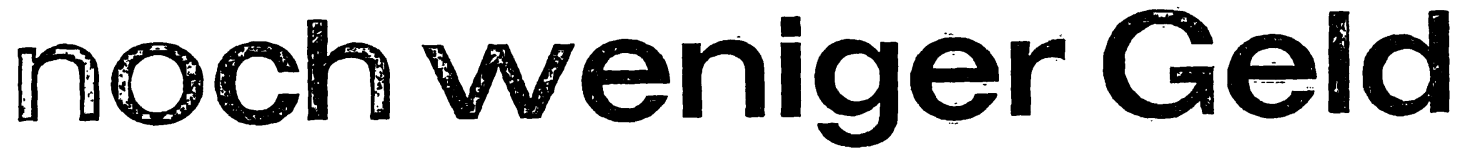
noch bessere

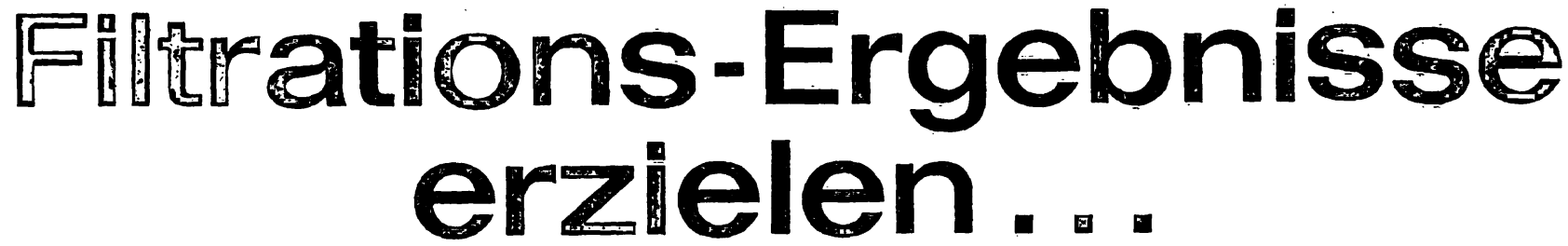

\section{Falls Sie nicht schon mit Sartorius-Membranfiltern arbeiten}

Sartorius-Membranfilter sind ein modernes und hochwertiges Filtermaterial aus Cellulosederivaten und anderen Polymerstoffen, das sich durch besondere Eigenschaften und strukturelle Gleichmäßigkeit von anderen Filtermedien grundlegend unterscheidet. Die hohe Durchflußleistung beruht auf einem Hohlraumvolumen von $85-65 \%$ entsprechend der Filterfeinheit und ca. $10^{8}$ Poren pro $\mathrm{cm}^{2}$. Sartorius-Membranfilter haben eine schaumartige, sehr gleichmäßige Struktur mit definierten Porengrößen von 10 Mikron bis $5 \mathrm{~nm}$.

Ihre Anwendung erstreckt sich auf die Anreicherung, Abscheidung und Trennung von Stoffen grobdisperser und kolloider Teilchen aus Flüssigkeiten oder Gasen durch Filtration. Weitere Anwendungsgebiete sind die Dialyse, die Osmose und die Elektrophorese.

Die Abtrennung erfolgt nach dem Siebeffekt: Alle Partikel, die größer sind als die mittlere Porengröße des Filters, werden zuverlässig auf der Oberfläche abgelagert. Konzentrationsänderungen durch Adsorption werden auf diese Weise vermieden. Sartorius-Membranfilter geben keine filtereigenen Bestandteile an das Filtrat ab und können im Filtrationsgerät autoklaviert werden. $\mathrm{Da}$ Sartorius-Membranfilter aus den verschiedensten Rohstoffen hergestellt werden, bietet sich die Möglichkeit, vom Labọrmaßstab bis zur großtechnischen Filtration jedem Anwendungsfall entsprechend das optimale Filter auszuwählen.

Genauso differenziert ist auch das dazugehörige Filtrationsgeräte-Programm: Es reicht von Spritzenvorsätzen über Filtrationsgeräte aus Glas, Edelstahl und Kunststoffen bis hin zu Mehrschichtenfiltrationsgeräten in Plattenbauweise.

Überzeugen Sie sich doch einmal selbst von den bemerkenswerten Eigenschaften unserer Filter und Geräte, indem Sie damit arbeiten. Wir senden Ihnen auf Anforderung gern jederzeit kostenlose Musterfilter und weiteres Informationsmaterial zu.

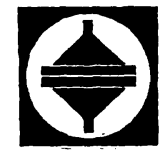

Sartorius Membranfilter

SARTORIUS-MEMBRANFILTER GMBH - 34 GOOTTINGEN - POSTFACH 142 
Kober-Farbstoffe ausführlich überprüft und fand, daß eine 50proz. Schwefelsäurekonzentration für eine optimale Bildung des zweiten Farbkomplexes vorhanden sein muß. Die Bildung des zweiten Farbstoffes konnte durch Anwesenheit von UV-Licht und längeres Erhitzen (12 Min.) verbessert werden.

Eine Konzentrierung und Reinigung der Kober-Farbstoffe gelang Irrrich (16) durch selektive Extraktion des Farbstoffes mit einer 2proz. Lösung von p-Nitrophenol in Chloroform: Äthylalkohol (99:1) oder besser durch eine 2proz. p-Nitrophenollösung in Acetylentetrabromid:Äthylalkohol (99:1) (26). Der auf diese Weise extrahierte Farbstoff zeigt eine hohe Empfindlichkeit besonders in der Fluorometrie. Das ITtrichsche Extraktionsprinzip in Kombination mit der Fluorometrie kommt besonders für Östriolbestimmungen in der Frühgravidität zur Anwendung (27).

Von Interesse ist die von Bachman (28) angegebene Farbreaktion, deren Spezifität für die Bestimmung von Östriol und 16-epi-Östriol erwiesen ist. Das Reagenz

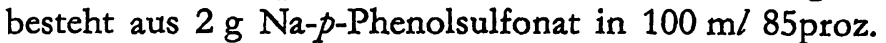
Phosphorsäure. Den Östriolproben werden $15 \mathrm{ml}$ des Reagenz zugegeben. Sie werden 9 Min. bei $150^{\circ}$ erhitzt. Dabei entsteht ein Violett-Rosa-Farbkomplex. Diese Farbreaktion wurde zur Bestimmung von Östriol in der Schwangerschaft bei EBERLEIN und Mitarbeitern (11), GreENe und Touchstone (14) und DaLe und Mitarbeitern (29) angewandt. Eine Zusammenstellung der Meßprinzipien enthält Tabelle 3.

Fremdstoffe, wie Phenolphthalein - und östrogenhaltige Substanzen können bei kolorimetrischer Messung durch Interferenz das Resultat verfälschen. Es sollte deshalb 2-3 Tage vor der Harnsammlung jegliche Medikation unterbleiben. Ebenfalls spielt der endogene Pigmentgehalt im Harn eine Rolle. Man kann ihn durch Einstellung auf eine möglichst konstante tägliche Harnmenge von etwa $1000 \mathrm{~m} l$ niedrig halten. Geringfügige Verunreinigungen lassen sich durch Anwendung der Allen-Korrektur weitgehend eliminieren. Ferner können durch das ITTRICHsche Extraktionsverfahren die meisten nicht durch Östrogene bedingten Chromogene entfernt werden.

Bei den fluorometrischen Methoden besteht ständig die Gefahr der Interferenz mit unspezifischen Substanzen aus biologischem Material. Hier ist deshalb in der Regel eine intensivere Reinigung der Extrakte erforderlich (30).

\section{Gaschromatographie}

Seit der Einführung der Gaschromatographie für Östriolbestimmungen im Schwangerenharn durch WotIz und Martin (31) liegen mit dieser Methode für die routinemäßige Bestimmung des Östriols nur vereinzelt Resultate vor. Yousem (32) und Touchistone (33) haben für die Bestimmung von freiem Östriol gaschromatographische Kurzmethoden entwickelt. Angaben zur Genauigkeit liegen für die beiden Methoden nicht vor. Mehr erfolgversprechend ist die Gaschromatographie von Östriolderivaten. Es eignen sich Acetate, da sie verhältnismäßig stabil sind $(19,21)$. Ferner haben sich Dimethylsilyl-Äther-Derivate des Östriols bewährt, die sich im Gegensatz zu freiem Östriol schnell von der Säule eluieren lassen (22, 23, 7). Die Reinigung der Extrakte ist für die gaschromatographischen Methoden ebenso aufwendig wie für kolorimetrische Methoden. Zusätzlich erfordert die Gaschromatographie viel Erfahrung und ist wesentlich teurer als die spektrophotometrischen Methoden. Die Vorstellung, daß grob gereinigte Harnextrakte auf die Gaschromatographie gegeben werden können, haben sich als falsch erwiesen. Im Gegenteil haben die Erfahrungen mit der Gaschromatographie gezeigt, $\mathrm{da} \beta$ diese Methode nur dann ausreichend genaue Werte liefert, wenn die Extrakte hoch gereinigt sind.

\section{Zuverlässigkeitskriterien für Östriolbestimmungs- methoden in der Schwangerschaft}

Nach Borth $(34,35)$ und Brown und Mitarbeitern (36) sind die wesentlichen Merkmale für die Zuverlässigkeit einer chemischen Hormonbestimmungsmethode Richtigkeit, Genauigkeit, Empfindlichkeit und Spezifität. Die drei ersten Kriterien wurden für die gebräuchlicheren Östriolbestimmungsmethoden geprüft, soweit entsprechende Daten angegeben waren (Tab. 4).

\section{Richtigkeit}

Die Richtigkeit einer Messung kennzeichnet nach der Borthschen Definition die Genauigkeit, mit der sie sich dem wahren Wert nähert. Sie kann für eine analytische Methode nur dann ausreichend bestimmt werden, wenn Zusatzversuche mit bekannten Mengen an reinem authentischem Material durchgeführt werden.

Optimale Bedingungen für die Richtigkeit einer Methode können durch routinemäßigen Zusatz von radioaktiv

Tab. 3

Meßprinzipien für Ostriolbestimmungen in der Schwangerschaft

Farbreaktionen:

1. Kober-Farbstoffe

a) nach BRown (2)

Ittrich-Farbstoffe

b) ITTRICH (16)

3. Bachman-Farbstoffe

BACHMAN (28) $\left.\begin{array}{lll}\text { (1. } 76 \% \mathrm{H}_{2} \mathrm{SO}_{4} ; & 2.55 \% \mathrm{H}_{2} \mathrm{SO}_{4} \\ \text { (1. } 76 \% \mathrm{H}_{2} \mathrm{SO}_{4} ; & \text { 2. } 50 \% \mathrm{H}_{2} \mathrm{SO}\end{array}\right)$

(Extraktion mit $2 \% p$-Nitrophenol in Chloroform oder Acetylentetrabromid)

$(2 \mathrm{~g} \mathrm{Na}$-p-Phenolsulfonat in $100 \mathrm{ml}$ 85 proz. Phosphorsäure) 
Tab. 4

Richtigkeit, Genauigkeit und Empfindlichkeit von Ostriolmethoden

\begin{tabular}{|c|c|c|c|c|c|c|}
\hline Methode & Östriolzusatz & $\begin{array}{c}\text { Richtigkeit } \\
\text { Wiederauffindur } \\
\% \neq s\end{array}$ & & Genauigkeit & $\left.n^{2}\right)$ & $\begin{array}{c}\text { Untere Grenze der } \\
\text { Empfindlichkeit } \\
\text { } \mathrm{g} / 24 \text { Stdn. } \\
\text { Harn }\end{array}$ \\
\hline $\begin{array}{l}\text { EBERLEIN und } \\
\text { Mitarbeiter (11) }\end{array}$ & 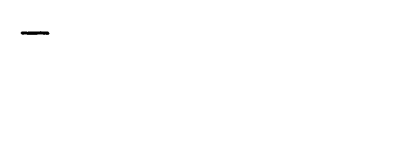 & - & , & $\begin{array}{l}\text { Doppelbestimmuingen mit } \\
1,2-9,0 \mu \mathrm{g} / 24 \text { Stdn.-Harn } \\
\mathrm{s} \pm 0,47 \mu \mathrm{g} \\
\text { mit } 11,1-42,7 \mu \mathrm{g} / 24 \mathrm{Stdn} .-\mathrm{Har} \\
\mathrm{s} \pm 1,17 \mu \mathrm{g}\end{array}$ & & $5-10$ \\
\hline CASSMER (18) & $10-20 \mu \mathrm{g}$ zum 24 Stdn.-Harn & $84 \pm 9,3$ & & $\begin{array}{l}\text { Doppelbestimmungen mit } \\
5-40 \mu \mathrm{g} / 24 \text { Stdn.-Harn } \\
\mathrm{s} \pm 1,24 \mu \mathrm{g}\end{array}$ & 32 & $5-10$ \\
\hline $\begin{array}{l}\text { PREEDY und } \\
\text { AITKEN (42) }\end{array}$ & $\begin{array}{l}2-12 \mu \mathrm{g} \text { zum } 24 \text { Stdn.-Harn } \\
13-30 \mu \mathrm{g} \text { zum } 24 \text { Stdn.-Harn } \\
31-50 \mu \mathrm{g} \text { zum } 24 \text { Stdn.-Harn } \\
1,3-11,9 \mu \mathrm{g} \mathrm{zu} 100 \mathrm{ml} \text { Plasma }\end{array}$ & $\begin{array}{l}75 \pm 14 \\
82 \pm 6 \\
88 \pm 5 \\
53-75 \%\end{array}$ & $\begin{array}{r}10 \\
6 \\
7 \\
9\end{array}$ & $\begin{array}{l}\text { Doppelbestimmungen mit } \\
2,9-12,3 \mu \mathrm{g} / 24 \text { Stdn.-Harn } \\
\mathrm{s} \pm 0,19 \mu \mathrm{g}\end{array}$ & & $\begin{array}{l}3-5 \\
0,1-0,5 \mu \mathrm{g} \text { pro } \\
100 \mathrm{ml} \text { Plasma }\end{array}$ \\
\hline $\begin{array}{l}\text { TAYLOR (40) } \\
\text { TAYLOR und } \\
\text { Mitarbeiter (44) }\end{array}$ & ( & etwa $85 \%$ & & s etwa $\pm 10 \%$ & & $20-50$ \\
\hline $\begin{array}{l}\text { KLOPPER und } \\
\text { WILSON (12) }\end{array}$ & $\begin{array}{c}\text { 2,4 } \mu \mathrm{g} \mathrm{zu} 10 \mathrm{ml} \text { Männerharn } \\
11,50 \mu \mathrm{g} \mathrm{zu} 5 \mathrm{ml} \text { Männerharn } \\
14,40 \mu \mathrm{g} \mathrm{zu} 1 \mathrm{ml} \text { Männerharn }\end{array}$ & $\begin{array}{l}74,3 \pm 12,5 \\
81,1 \pm 4,1 \\
87,7 \pm 5,8\end{array}$ & $\begin{array}{l}16 \\
16 \\
16\end{array}$ & $\begin{array}{l}\mathrm{s} \pm 17,3 \%(2,41 \mu \mathrm{g}) \\
\pm 5,1 \%(11,50 \mu \mathrm{g}) \\
\pm 6,3 \%(14,40 \mu \mathrm{g})\end{array}$ & . & $20-50$ \\
\hline $\begin{array}{l}\text { STOA und } \\
\text { THORSEN (27) }\end{array}$ & $0,05-0,1 \mu \mathrm{g}$ zu $25 \mathrm{ml}$ Harn & $84-94$ & & $\begin{array}{l}\text { Doppelbestimmungen mit } \\
0,3-0,5 \mu \mathrm{g} \\
\mathrm{s} \pm 0,06 \mu \mathrm{g} \\
\text { mit } 0,51-1,0 \mu \mathrm{g} \\
\mathrm{s} \pm 0,08 \mu \mathrm{g} \\
\text { mit } 1,01-3,0 \mu \mathrm{g} \\
\mathrm{s} \pm 0,18 \mu \mathrm{g}\end{array}$ & $\begin{array}{l}11 \\
16 \\
23\end{array}$ & $20-50$ \\
\hline $\begin{array}{l}\text { BROWN und } \\
\text { COYLE (13) } \\
\text { (3 Methoden) }\end{array}$ & $\begin{array}{l}5 \mu \mathrm{g} \mathrm{zu} 20 \mathrm{ml} \text { verdünntem } \\
\text { oder unverdünntem Harn }\end{array}$ & $79 \pm 4,6$ & 23 & $\begin{array}{l}\text { Doppelbestimmungen mit } \\
0,1-1 \mathrm{mg} / 24 \text { Stdn.-Harn } \\
\mathrm{s} \pm 6,7 \% \\
1 \pm 20 \mu \mathrm{g} / 24 \text { Stdn.-Harn } \\
\mathrm{s} \pm 5,7 \%\end{array}$ & $\begin{array}{l}19 \\
39\end{array}$ & $70 \div 100$ \\
\hline & $\begin{array}{l}5 \mu \mathrm{g} \text { zu } 20 \mathrm{ml} \text { verdünntem } \\
\text { oder unverdünntem Harn }\end{array}$ & $82 \pm 3,9$ & 23 & 一 & & $300-500$ \\
\hline & $\begin{array}{l}5 \mu \mathrm{g} \text { zu } 20 \mathrm{ml} \text { verdünntem } \\
\text { oder unverdünntem Harn }\end{array}$ & $85 \pm 6,1$ & 36 & 一 & & $300-500$ \\
\hline BELING (16) & $\begin{array}{l}0,5 \mu \mathrm{g} \text { Ostriol-16, } 17- \\
\text { glucosiduronat } \\
5 \mu \mathrm{g} \text { Ostriol-3-sulfat } \\
0,5-20 \mu \mathrm{g} \text { Ostriol-3- } \\
\text { glucosiduronat jeweils } \\
\text { zu } 5 \mathrm{ml} \text { Männerharn }\end{array}$ & $\begin{array}{l}70 \pm 6,4 \\
78 \pm 7,0 \\
78,7(6)\end{array}$ & $\begin{array}{r}10 \\
8\end{array}$ & $\begin{array}{l}\text { Wiederholungsbestimmungen } \\
\text { mit } 0,72-23,4 \mathrm{mg} \text { Ostriol } \\
\text { pro } 24 \text { Stdn.-Harn } \\
\mathrm{s} \pm 6 \%\end{array}$ & 114 & $100-300$ \\
\hline $\begin{array}{l}\text { GREEN und } \\
\text { TOUCHSTONE (14) }\end{array}$ & $60 \mu \mathrm{g}$ Östriol zu Wasser & $54 \pm 10$ & 24 & - & & $20-50$ \\
\hline $\begin{array}{l}\text { ADLERCREUTZ und } \\
\text { LUUKKAINEN }(7,47)\end{array}$ & ansog & 一 & & $\begin{array}{l}\text { Doppelbestimmungen mit } \\
0,22-4,94 \mathrm{mg} / 24 \text { Stdn.-Harn } \\
\mathrm{s} \pm 0,12 \mathrm{mg} \\
\text { mit 7,57-56,8 mg/24 Stdn.-Ha } \\
\mathrm{s} \pm 0,32 \mathrm{mg}\end{array}$ & $\begin{array}{l}15 \\
\mathrm{rn} \\
14\end{array}$ & $100-200$ \\
\hline $\begin{array}{l}\text { NAcHTIGALL und } \\
\text { Mitarbeiter (38) }\end{array}$ & $\begin{array}{l}\left.6000 \text { Imp./Min. Östriol--[ }{ }^{8} \mathrm{H}\right]- \\
\text { Konjugate zu } \mathrm{i} \mathrm{ml} \\
\text { Schwangerenplasma }\end{array}$ & $55-88 \%$ & & $\begin{array}{l}\text { Wiederholungsversuche mit } \\
\text { Spätschwangerenplasma } \\
20,7 \pm 2,5 \mu \mathrm{g} / 100 \mathrm{ml}\end{array}$ & 27 & 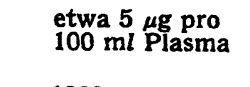 \\
\hline $\begin{array}{l}\text { SCHINDLER und } \\
\text { HERRMANN (22) }\end{array}$ & $\begin{array}{l}\text { 5491 Imp./Min. Ostriol- } \\
{\left[6,7-\mathrm{H}^{3}\right] \text { zu Männerharn }}\end{array}$ & $71,6(5)$ & & $\begin{array}{l}\text { Wiederholungsversuche mit } \\
\text { Spätschwangerenharn } \\
\mathrm{s} \pm 1,5 \mathrm{mg} / 24 \text { Stdn.-Harn }\end{array}$ & & 1000 \\
\hline $\begin{array}{l}\text { NILSSON und } \\
\text { BENGTSSON }(21)\end{array}$ & $\begin{array}{l}50-125 \mu \mathrm{g} \mathrm{zu} 200 \mathrm{ml} \\
\text { Männerharn }\end{array}$ & $74,8 \pm 3,7$ & 8 & $\begin{array}{l}\text { Doppelbestimmungen mit } \\
0,268-4,21 \mathrm{mg} / 24 \mathrm{Stdn} \text {-Harn } \\
\mathrm{s} \pm 3,6 \%\end{array}$ & 4 & $100-500$ \\
\hline $\begin{array}{l}\text { RUNNEBAUM und } \\
\text { Mitarbeiter }(48) \\
\text { (nach 1. c. (37), (46)) }\end{array}$ & $\begin{array}{l}9,6 \mu \mathrm{g} \mathrm{zu} 5 \mathrm{ml} \text { Harn } \\
\text { auf } 20 \mathrm{ml} \text { verdünnt } \\
9,6 \mu \mathrm{g} \mathrm{zu} 20 \mathrm{ml} \text { Wasser }\end{array}$ & $\begin{array}{l}5,6 \pm 0,53 \mu \mathrm{g} \\
7,7 \pm 0,14 \mu \mathrm{g}\end{array}$ & $\begin{array}{l}10 \\
10\end{array}$ & $\begin{array}{l}\text { Doppelbestimmungen mit } \\
1-5 \mathrm{mg} / 24 \text { Stdn.-Harn } \\
\text { s } \pm 0,26 \\
\text { mit } 5-20 \mathrm{mg} / 24 \text { Stdn.-Harn } \\
\text { s } \pm 0,25 \mathrm{mg}\end{array}$ & $\begin{array}{l}30 \\
30\end{array}$ & 1000 \\
\hline
\end{tabular}

1) Anzahl der Doppelbestimmungen

markierten Steroiden erzielt werden. Allerdings ist diese Forderung für klinische Routinemethoden in der Regel nicht praktikabel, da häufig keine entsprechenden Geräte für die Messung der Radioaktivität zur Verfügung stehen. Es kann somit durch Zusatzversuche mit bekannten Mengen an Östriol die mittlere Wiederauffindung mit der Standardabweichung ermittelt werden. Von mehr als 20 Östriolbestimmungsmethoden, die speziell in der Schwangerschaft zur Anwendung kommen, fanden sich in nur etwa der Hälfte der Methoden ausreichende Daten zur Richtigkeit. Die Wiederauffindungen für Östriol bewegen sich zwischen 55 und 85\%. Wenn für die Verluste während der Aufarbeitung nicht korrigiert wird, wie es in den meisten Östriolmethoden der Fall ist, so werden Östriolwerte verschiedener Laboratorien kaum miteinander vergleichbar sein.

\section{Genauigkeit}

Die Genauigkeit einer Methode ergibt sich aus der Reproduzierbarkeit der Messungen. Sie kann einmal durch Wiederholungsversuche bestimmt werden. Ein weiteres $\mathrm{Maß}$ für die Genauigkeit ist die Standardabweichung aus den Differenzen der Ergebnisse von Doppelbestimmungen. Die 2. Methode eignet sich besonders für Routinemethoden, weil dabei die Genauigkeit über einen längeren Zeitraum ermittelt werden 
kann, die bei der Handhabung der biologischen Proben erreicht wird. Die Genauigkeit ist ebenfalls für etwa die Hälfte der Östriolbestimmungsmethoden ausreichend bekannt. In den letzten 2 Trimestern der Schwangerschaft sind Methoden von KLOPpER und Wirson (12), BeLING (6) und von Brown und Coyle (13) ausreichend genau, allerdings'zum Teil mit einem zu großen Arbeitsaufwand verbunden. Nur unzureichende oder keine Genauigkeitskriterien liegen für die meisten gaschromatographischen Methoden vor $(31,32,33,20,23)$. Die etwas aufwendigen gaschromatographischen Methoden von Adlercreutz und LuUkKainen (7) und Nilsson und Bengtsson (21) machen eine Ausnahme.

\section{Empfindlichkeit}

Die Empfindlichkeit einer analytischen Methode ist durch die geringste Menge einer reinen Substanz bestimmt, die mit der Methode noch quantitativ erfaßt werden kann. Es muß allerdings unterschieden werden zwischen der geringsten Menge reinen Östriols, das noch quantitativ erfaßt werden kann und zwischen der geringsten Östriolmenge, die mit ausreichender Richtigkeit und Genauigkeit aus biologischem Material isoliert gemessen werden kann. $\mathrm{Da}$ die Östriolkonzentrationen im Harn im Verlauf der Schwangerschaft um den Faktor 1000 und mehr ansteigen, können naturgemäß Methoden mit sehr unterschiedlicher Empfindlichkeit zur Anwendung kommen. Die untere Grenze der Empfindlichkeit liegt für einige aufwendige Östriolmethoden (Modifikationen nach BRown (2) und BAULD (3)) bei $5-10 \mu \mathrm{g}$ im 24 Stundenharn. Für klinisch anwendbare Östriolmethoden in der Schwangerschaft ist die untere Grenze der Empfindlichkeit häufig nicht exakt bekannt, so daß bei Bestimmungen in der Frühgravidität mit einer größeren Variabilität der Werte durch methodische Fehler gerechnet werden muß.

\section{Spezifität}

Wichtige Schlüsse für die Spezifität einer Methode können zunächst aus den Prinzipien gezogen werden, auf denen die Methode bzw. ihre verschiedenen Arbeitsgänge beruhen. Mit einer angemessenen Spezifität einer mikroanalytischen Methode zur Bestimmung von
Östriol kann man rechnen, wenn eine ausreichende chromatographische Trennung des Östriols von den Verunreinigungen und von den anderen Steroiden erfolgt. Die Spezifität einer Methode ist immer dann besonders wichtig, wenn nur geringe Mengen der zu messenden Substanz im biologischen Material vorhanden sind. Bei Östriolbestimmungen in der Schwangerschaft mit hohen Steroidkonzentrationen zeigt sich, daß selbst bei den Methoden, die keine Derivatbildung des Östriols oder eine Chromatographie beinhalten, an dem Endprodukt die Charakteristika des Östriols nachweisbar waren (8).

\section{Kriterien für die Wahl einer Östriolmethode}

Bei der Auswahl einer Östriolmethode für ein konventionelles Laboratorium sind Zeit und Materialaufwand, die für eine Bestimmung erforderlich sind, entscheidend. Eine Schnellmethode dieser Art sollte jedoch ebenfalls ausreichend spezifisch und genau sein. Aus diesen Forderungen geht hervor, daß im Verlauf der Schwangerschaft wie bereits erwähnt, Östriolmethoden mit unterschiedlichem Empfindlichkeitsbereich angewandt werden müssen.

Östriolbestimmungsmethoden, die wenigstens zum Teil eine Bewertung für einen bestimmten quantitativen Bereich zulassen, sind in Tabelle 5 ersichtlich. Die Wiederauffindungen bewegen sich zwischen 54 und $85 \%$. Da die Werte in der Regel nicht für Verluste korrigiert werden, wird hieraus verständlich, $\mathrm{da} B$ jedes Laboratorium eine eigene Normalkurve zu Vergleichszwecken braucht. Die Genauigkeit beträgt für die genannten Methoden zwischen 6 und 10\%. Allerdings schwanken die quantitativen Bereiche für die angegebenen Genauigkeitswerte zwischen 0,1 und $15 \mathrm{mg}$ pro 24-Stundenharn.

Es erhebt sich die Frage, wieviele Bestimmungen innerhalb welcher Zeit in einer bestimmten Schwangerschaftsphase durchführbar sind. Mit der Methode nach BeLING (6) sind beispielsweise etwa ab der 14. Schwangerschaftswoche bei ausreichender Genauigkeit 20-30 Bestimmungen pro Tag möglich. Im Vergleich dazu läßt die Methode nach FRANDSEN und STAKEMANN (37) ab der 20. Schwangerschaftswoche in $4-5 \mathrm{Stdn}$. $10-20 \mathrm{Be}-$

Tab. 5

Vergleich zwischen Zuverlässigkeit und Arbeitsaufwand einiger Ostriolbestimmungsmethoden

\begin{tabular}{|c|c|c|c|c|c|c|c|c|}
\hline $\begin{array}{l}\text { Zuverlässigkeits- } \\
\text { kriterien) }\end{array}$ & $\begin{array}{c}\text { TAYLOR } \\
\text { und } \\
\text { Mitarbeiter } \\
(40)\end{array}$ & $\begin{array}{c}\text { BELING } \\
\text { (6) }\end{array}$ & $\begin{array}{c}\text { GREENE } \\
\text { und } \\
\text { TouchSTONE } \\
(14)\end{array}$ & $\begin{array}{c}\text { RUNNEBAUM }{ }^{2} \text { ) } \\
\text { und } \\
\text { Mitarbeiter } \\
\text { (48) }\end{array}$ & $\begin{array}{l}\text { SCHINDLER } \\
\text { und } \\
\text { HERRMANN } \\
\text { (22) }\end{array}$ & $\begin{array}{l}\text { KLOPPER und } \\
\text { STEPHENSON } \\
\text { (43) }\end{array}$ & $\begin{array}{c}\text { BROWN und } \\
\text { COYLE } \\
(13)\end{array}$ & $\begin{array}{c}\text { BROWN } \\
\text { und } \\
\text { Mitarbeiter } \\
\text { (5) }\end{array}$ \\
\hline $\begin{array}{l}\text { Richtigkeit } \\
\%(\overline{\mathrm{x}} \pm \mathrm{s})\end{array}$ & etwa 85 & $78 \pm 7$ & $54 \pm 10$ & $58 \pm 9$ & 71,6 & $81 \pm 4$ & $79 \pm 4,6$ & $75 \pm 3$ \\
\hline $\begin{array}{l}\text { Genauigkeit VK (\%) } \\
\text { Empfindlichkeits- } \\
\text { bereich mg Ostriol/ } \\
24 \text { Stdn. }\end{array}$ & $\begin{array}{c}\text { etwa } \pm 10 \\
-\end{array}$ & $\begin{array}{c} \pm 6 \\
0,7-23\end{array}$ & - & $\begin{array}{l} \pm 10 \\
1-5\end{array}$ & $\begin{array}{l} \pm 5-10 \\
15-40\end{array}$ & $\stackrel{ \pm}{ \pm 0}$ & $\begin{array}{c} \pm 6,7 \\
0,1-1,0\end{array}$ & $\begin{array}{l} \pm 5,6 \\
1-10\end{array}$ \\
\hline $\begin{array}{l}\text { Untere Grenze der } \\
\text { Empfindlichkeit } \\
\text { (mg Óstriol/24 Stdn.) }\end{array}$ & $0,02-0,05$ & $0,1-0,3$ & $0,02-0,05$ & 1,0 & 1,0 & $0,05-0,1$ & 0,1 & - \\
\hline $\begin{array}{l}\text { Anwendung: } \\
\text { Stunden } \\
\text { Bestimmungen }\end{array}$ & $\begin{array}{r}8 \\
10\end{array}$ & ${ }_{20-30}^{\left.8(+16)^{2}\right)}$ & $\begin{array}{l}8 \\
8\end{array}$ & $\begin{array}{c}4-5 \\
10-20\end{array}$ & $6-8$ & $\begin{array}{l}8 \\
6\end{array}$ & $\begin{array}{l}6 \\
8\end{array}$ & $12^{3 / 2}$ \\
\hline
\end{tabular}

2) Methode nach FRANDSEN und STAKEMANN (37), Hydrolyse mit 15 Vol. \% HCl, 1 Std. erhitzen.

2) Die angegebene Zuverlăssigkeit bezieht sich auf die genannten quantitativen Bereiche (mg Ostriol/24 Stdn.)

3) Dauer der enzymatischen Hydrolyse 16 Stdn. 
stimmungen zu. Für die ersten Wochen in der Schwangerschaft kommt z. B. die Methode nach KLOPPER und Wirson (12) in Frage, mit der 6 Bestimrnungen pro Tag durchgeführt werden können, oder ab dem 2.-3. Schwangerschaftsmonat die Methode nach BRown und Coyre (13), mit der sich 8 Bestimmungen in $6 \mathrm{Stdn}$. durchführen lassen. Die Methode nach BRown und Mitarbeitern (5) erfaßt die Gesamtöstrogene und soll hier nur vergleichsweise angeführt werden. Es können durch Automatisierung der Extraktionsschritte $12 \mathrm{Be}$ stimmungen in $3 \frac{1}{2} \mathrm{Stdn}$. durchgeführt werden. Eine Begründung für die unterschiedliche Empfindlichkeit der einzelnen Methoden gibt Abbildung 3.

Aus Abbildung 3 wird deutlich, daß die meisten Autoren entweder eine Methylierung oder eine Säulenchromatographie für eine Östriolbestimmung erforderlich halten. Die Methoden, die sowohl eine Methylierung und eine Säulenchromatographie einschließen, sind gewöhnlich für Östriolbestimmungen im ersten Drittel der Schwangerschaft ausreichend empfindlich und genau.

Die vorgelegten Daten zeigen, daß für jede Phase der Schwangerschaft Methoden zur Verfügung. stehen, die mit ausreichender Zuverlässigkeit den Östriolgehalt im 24-Stundenharn erfassen könnèn.

In Abbildung 4 sind die verschiedenen Normbereiche einiger hier angeführter Östriolbestimmungsmethoden einander gegenübergestellt. Bei den verschiedenen Methoden wird eine deutliche Streuung des Normbereiches sichtbar.

Die bisher vorliegenden Werte für Östriol im peripheren Blut schwangerer Frauen lassen gegenwärtig noch keine Schlüsse zu, ob solche Bestimmungen im Plasma einen zuverlässigen Indikator für den Zustand von Fetus und Plàcenta darstellen $(38,39)$.

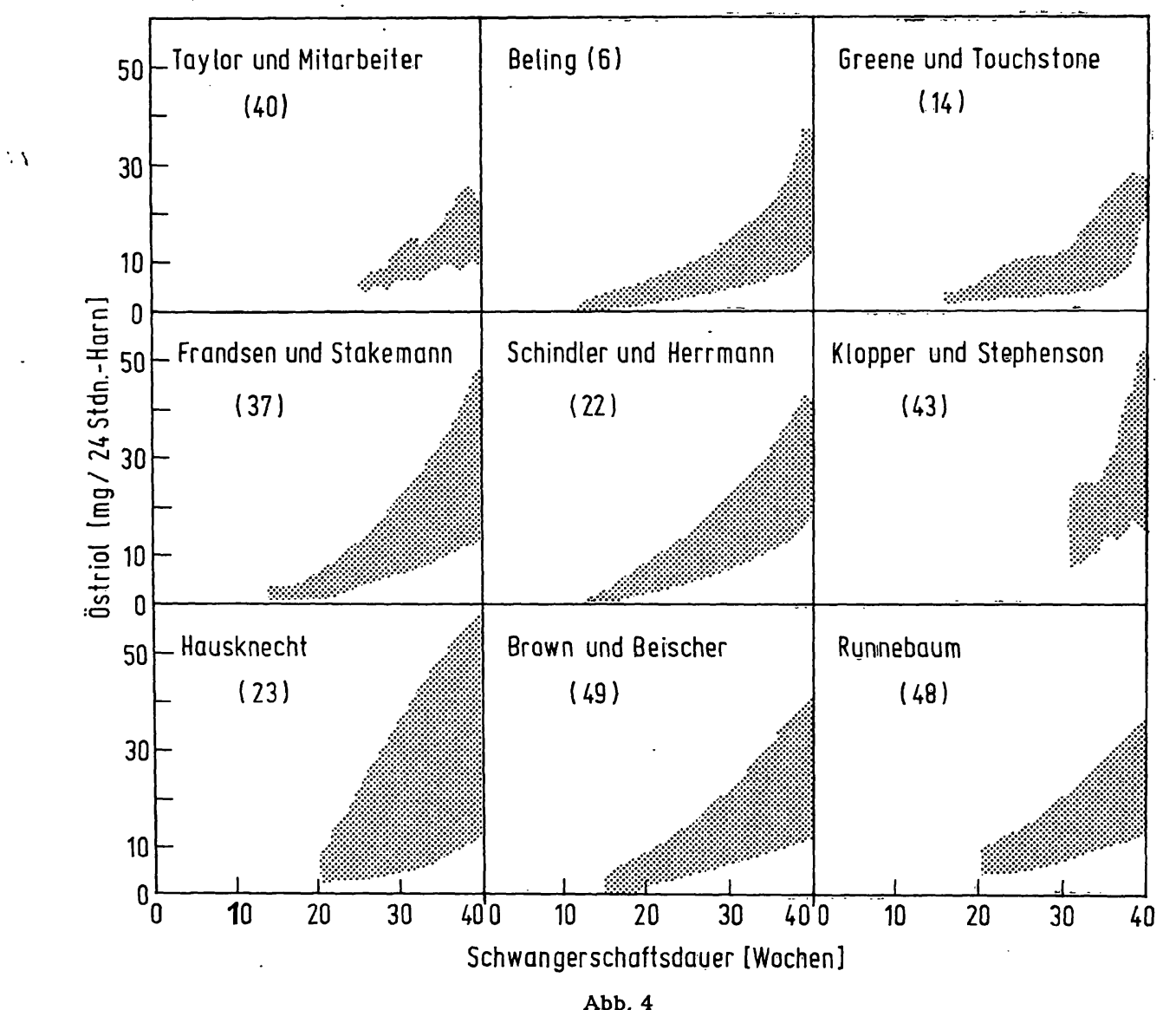

Normalkurven verschiedener Laboratorien für die Ostriolausscheidung im Harn während der Schwangerschaft

\section{Literatur}

1. Stevenson, M. F. und G. F. Marrian, Biochem. J. 41, 507 (1947). - 2. Brown, J. B., Biochem. J., 60, 185 (1955). - 3. BAULD, W. S., Biochem. J., 63, London, 488 (1956). - 4. BROWN, J. B. und H. A. F. BlaIr, J. Endocr. 17, 411 (1958). - 5. Brown, J. B., S. C. McLeod, C. Macnaugritan, M. A. Smith und B. Smith, J. Endocr. 42, 5 (1968). - 6. Beling, C. G., Acta endocr., K'hon Suppl. 79 (1963). - 7. AdLercreutz, H. und T. LuUKKAINEN, Gas phase chromatographic methods for estrogens in biological fluids. In: Monogr. on Endocrinology, (eds. K. B. Eik-Nes and E. C. Horning) Vol. II, p. 72. Springer-Verlag, Berlin-Heidelberg-New York (1968). - 8. FRANDSEN, V. A.,
The excretion of oestriol in normal human pregnancy, Copenhagen, Munksgaard (1963). - 9. Hobkirk, R., A. AlfHerm ünd S. BuGGE, J. Clin. Endocr., Springfield 19, 1352 (1959). - 10. Frandsen, V. A., Acta endocr., K'hrn 50, 418 (1965). - 11. EarrLeIN, W. R., A. M. Bongrovanni und C. M. Francrs, J. Clin. Endocr., Springfield 18, 1274 (1958). - 12. Klopper, A. I. und G. R. WILSON, J. Obstetr. Gynaec. Brit. Empire 69, 533 (1962). - 13. Brown, J. B. und M. G. CoxLE, J. Obstetr. Gyncac. Brit.Empire 70,219 (1963). 14. Greene, J. W. Jr. und J. C. Touchstone, Am. J. Obstetr. Gynec., 85, 1 (1963). - 15. Diczanlusy, E. und A. Westiman, Acta endocr., K'hvn 21, 321 (1956). - 16. IrrRIĆH, G., Hoppe-Seylexs Z. 


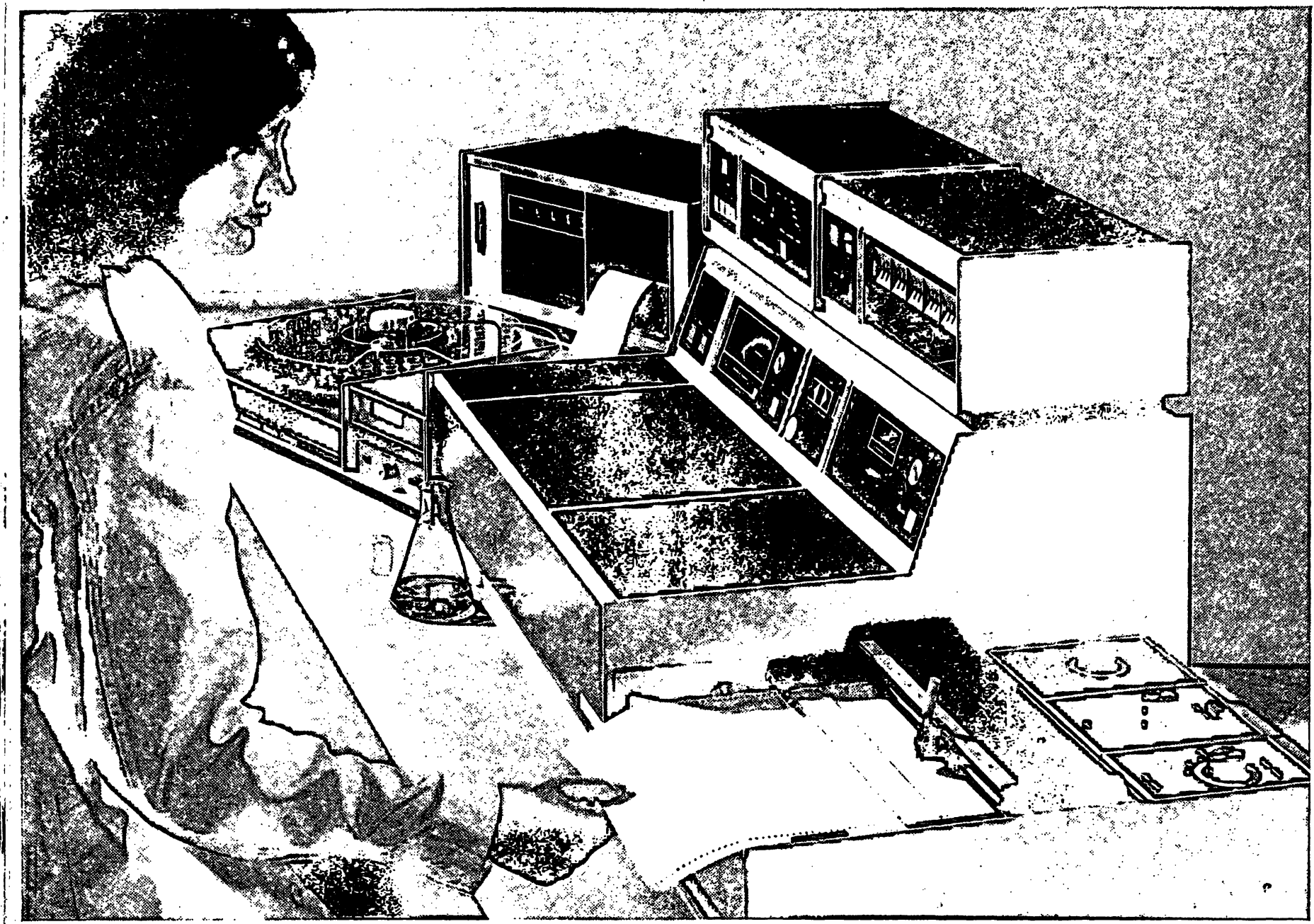

Sie analysieren vielseitiger, schneller, bequemer und erzielen genauere Ergebnisse mit dem UV-Spektralphotometer SP 1800 - eine Neuentwicklung aus dem Programm PYE UNICAM

\section{damit ist lhr Amalysenproblem gelöst}

1. Das Doppelstrahl-Spektralphotometer für den ultravioletten und sichtbaren Bereich hat die hohe photometrische Genauigkeit von $\pm 0,002$ E. Es wird als Grundgerät eingesetzt mit direkter Extinktionsanzeige auf eingebautem Zeigerinstrument mit 4 Extinktionsskalen $(0-0,2 \mathrm{E} ; 0-0,5 \mathrm{E} ; 0-1,0 \mathrm{E}$ und $0-2,0 \mathrm{E})$; umschaltbar für Konzentrationsanzeige; hochauflösendem Gitter-Monochroma: tor; großem Probenraum und zweiter Probenstelle; automatischem Lampenwechsel.

Als vollautomatisches Spektralphoto-
meter-System bietet es Ihnen durch mehrere Zusatz-Einheiten weitere Vorteile:

Spektrendarstellung - komprimierte und gedehnte Spektren - durch synchronisierte Registrierung auf dem Schreiber AR 25; digitale Datenausgabe; wiederholte Registrierung eines Wellenlängenbereiches sowie bei mehreren automatisch vorwählbaren Wellenlängen; Mehrkanalbetrieb für kinetische Untersuchungen; automatischer Probenwechsler; besondere Schaltungen erlauben das Programmieren von wiederholten, ver- zögerten oder ferngesteuerten Messungen mehrerer Proben.

Ausführliches Informationsmaterial liegt für Sie bereit. Bitte fordern Sie es an.

Philips Elektronik Industrie $\mathrm{GmbH}$ 2000 Hamburg $63 \cdot$ Röntgenstraße 22 Telefon (0411) 501031

Büros in: Berlin Tel. (0311) 245908 , Düsseldorf Tel. (0211) 3460 51, Dortmund Tol. (0231) 41961 , Frankfurt Tel. (0611) 79131 . Hannover Tol. (0511) 16601, München Tel. (0811) 76791 , Stuttgart-Fellbach Tel. (0711) 589081 , Bielefeld Tel.
(0521) 230 81, Bremen Tel. (0421) 3100 41, Nürnberg Tel. (0911) 464763 .

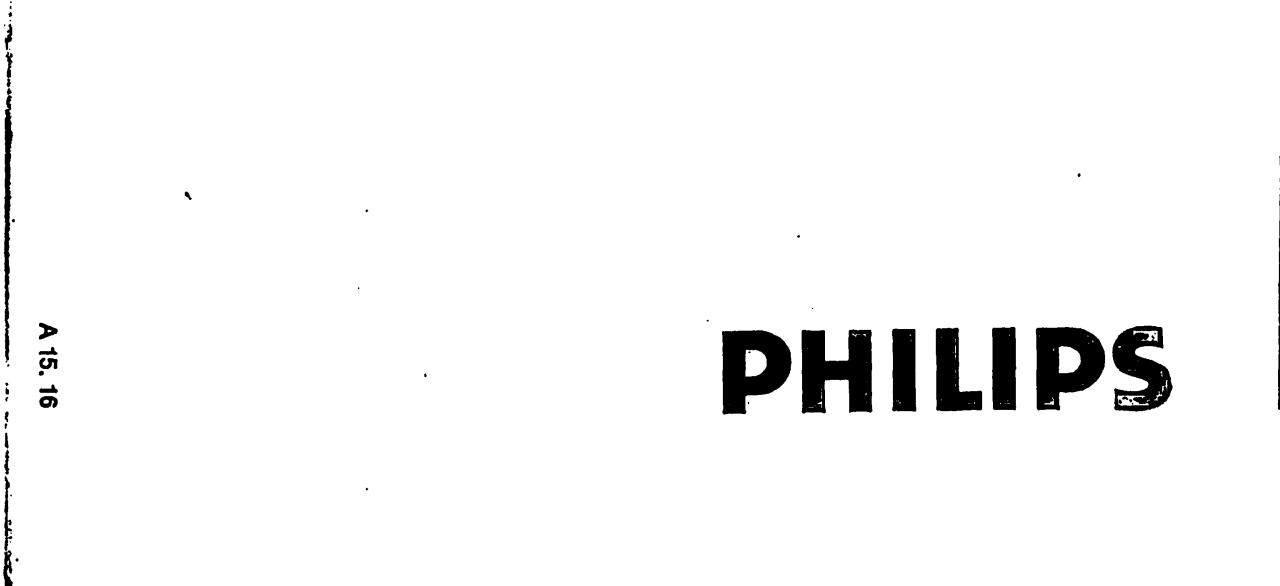
V-Spektralphotometer SP 1800 und bitten um

7 Zusendung ausführlicher Unterlagen ein Angebo

eine unverbindliche Vorführung

Gewünschtes bitte ankreuzen oder ergänzen 


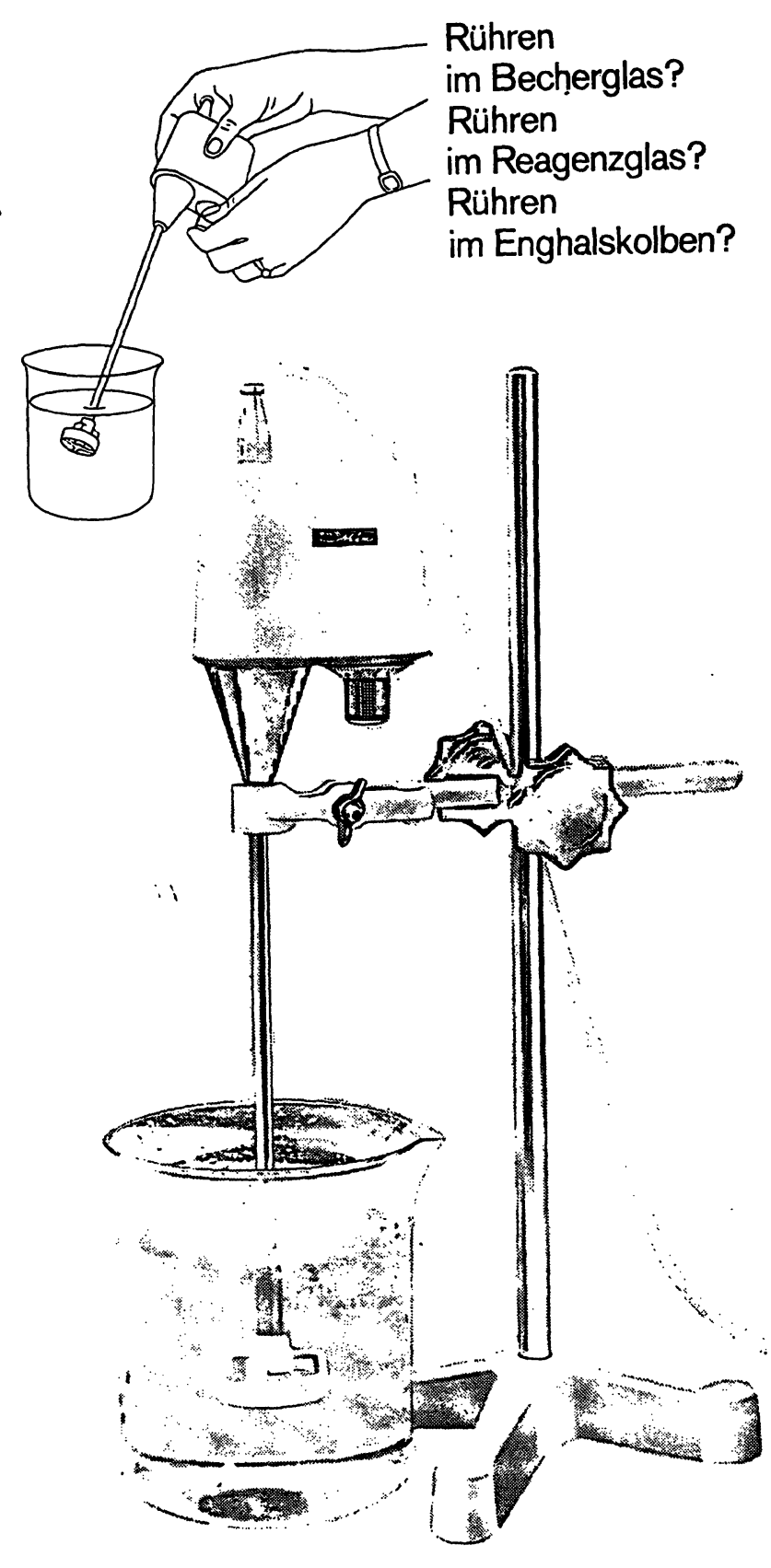

IKA-RM10 Mix

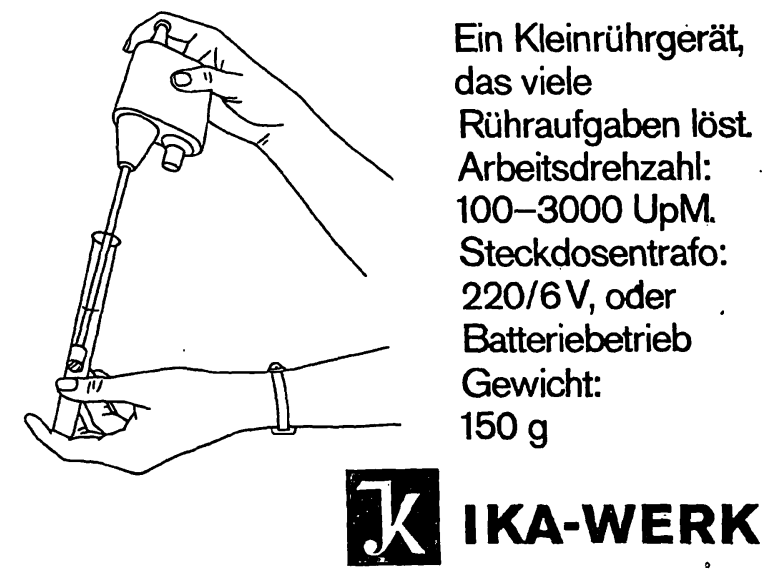

Janke \& Kunkel KG. 7813 Staufen i. Br. Postfach 44 · Tel. (07633) 5294
ARBEITSMETHODEN

DER MODERNEN NATURWISSENSCHAFTEN

Herausgegeben von Prof. Dr. KuRT FisCHBECK

\section{KonRad Dorfner \\ Ionenaustäuscher}

\section{Auflage}

Mit 100 Abbildungen, 27 Tabellen im Text und 1 Tabellenanhang (mit 19 Tabellen). Oktav. XII, 320 Seiten. 1970. Plastikeinband DM 58,-

Nach der erweiterten Einführung wird eine ausführlichere Darstellung der verschiedenen Ionenaustauschertypen sowie ihrer Eigenschaften und Prüfmethoden gegeben. Besonders über die Verwendung der Ionenaustauscher in der Technik wird im einzelnen berichtet, um den Neuentwicklungen gerecht zu werden. So sind die rechnerische Behandlung und die speziellen Verfahren des Festbettverfahtrens, die neuesten Entwicklungen der kontinuierlichen Verfahren, die Wasseraufbereitung mit Ionenaustauschern und die Verwendung der Ionenaustauscher zur Abwasserreinigung, Metallgewinnung, Zucketherstellung und sonstiger technischer Anwendungen in dem vorgegebenen Rahmen so umfassend wie möglich dargestellt worden. Die übrigen Kapitel wurden nach den neuesten Ërgebnissen durchgearbeitet, verbeșsert und ergänzt.

JoHANNES FLÜGGE

\section{Grundlagen der Polarimetrie}

Gerätekunde und Meßtechnik

Oktav. Mit 72 Abbildungen und 28 Tabellen. XII, 159 Seiten. 1970. Plastikeinband DM 48,-

Wurde die Polarimetrie bereits seit langem als analytisches Verfahren, z. B. in Zuckerfabriken und in Betrieben der pharmazeutischen Chemie, angewandt, so hat sie sich in neuerer Zeit auch in der Erforschung von Molekülstrukturen als aufschlußreich exwiesen, besonders seitdem es automatische und Spektralpolarimeter bis ins Ultraviolett gibt. Das vorliegende Werk informient über Grundlagen, Meßtechnik und moderne Geräte dieser optischen Methode und berücksichtigt ihren Stand bis in die jüngste Zeit, wobei neben der Analytik auch die Bestimmung der Rotationsdispersion, der magneto-optischen Drehung des Lichts und der Elliptizität, wie sie bei Zirkulardichroismus auftritt, besprochen werden. Photoelektrische Polarimeter und Saccharimeter werden ausführlich behandelt.

Walter de Gruyter \& Co $\cdot$ Berlin 
physiol. Chem. 3121 (1958). - 17. ENGEL, E. E., W. R. Jr. Slaunwhite, P. Carter und I. I. Nathanson, J. biol. Chemistry 185, 255 (1950). - 18. Cassmer, O., Acta endocr., K'hvn Suppl. 45, 25 (1959). - 19. Wotiz, H. H. und S. J. Clark, Gas Chromatography in the Analysis of Steroid Hormons. Plenum Press, New York (1966). - 20. Bengtsson, L. Ph. und B. Forsgren, Acta obstetr. gynec. Scand. 45, 155 (1966). - 21. Nirsson, I. und L. Pr. Bengtsson, Acta obstetr. gynec. Scand. 47, 213 (1968). - 22. Schindler, A. E. und W. L. Herrmann, Gynaecologia (Basel) 161, 446 (1966). - 23. Hausknecht, R. U., Obstetr. Gynec. Guide, Wash. 30, 639 (1967). - 24. Kober, S., Biochem. Z, 239, 209 (1931). - 25. Nocke, W., Biochem. J. 78, 593 (1961). 26. Irtrich, G., Acta endocr., K'hvn 35, 34 (1960). - 27. StoA, K. F. und T. Thorsen, Acta endocr. K'hrn 41, 481 (1962). 28. BachmanN, C. J., biol. Chemistry 131, 463 (1939). 29. Dale, E., J. W. Greene, und J. L. Duhring, Amer. J. Obstetr. Gynec. 92, 112 (1965). - 30. Scholler, R., P. LeYMarie, M. Heron und M. F. JAYLE, Acta endocr. K'hvn 107, 7 (1966). 31. Worrz, H. H. und H. F. Martin, Fed. Proc. 20, 99 (1961). 32. Yousem, H. L., Amer. J. Obstetr. Gynec. 88, 375 (1964). 33. Touchstone, J. C., J. Gaschromatography 170 (1964). 34. Borth, R., Ciba Found Collog. Endocr. 2, 45 (1952). - 35 Borth, R., Vitamines and Hormones 15, 259 (1957). - 36. Brown, J. B., R. D. Bulbrook, und F. C. Greenwood, J. Endocr.
16, 41 (1957). - 37. Frandsen, V. A. und G. Stakemann, Acta endocr. K'hvn 44, 183 (1963). - 38. NAchtigall, L., M. BASSETt, U. Hogsander, S. Slagle und M. Levitz, J. Clin. Endocr., Springfield 26, 941 (1966). - 39. Selinger, M. und M. Levitz, J. Clin. Endocr., Springfield (1969) 995. - 40. TArzor, E. S., P. D. Bruns, J. W. Duncan und V. E. Drose, Amer. J. Obstetr. Gynec. 81, 625 (1961). - 41. Woriz, H. H. und H. F. Martin, Analytic. Biochem. 3, 97 (1962). - 42. PREEDY, R. K. und E. H. Artken, J. biol. Chemistry. 236, 1300 (1961). - 43. Klopper, A. I. und R. Stephenson, J. Obstetr. Gynaec. Brit. Empire 69, 28 (1966). - 44. Taylor, E. S., A. Hassner, P. D. Bruns und V. E. Drose, Amer. J. Obstetr. Gynec. 85, 10 (1963). - 45. Touchstone J. C., J. W. Jr. Greene, R. C. McElroy und T. Murawec, Biochemistry USA 2, 653 (1963). - 46. FrandSEN, V. A. und G. Stakemann, Acta endocr. K'hvn 44, 196 (1963). - 47. AdlerCReutz, H. und T. LuUkKarnen, Determination of urinary estrogens by gas chromatography. In: Gas chromatography of steroids in biological fluids, (ed. M. B. Lipsett). Plenum Press, p. 215. New York (1965). - 48. RunNebaum, B., K. HolzmanN und J.ZANDER, unveröffentlichte Resultate. 49. Brown, J. B. und N. A. Beiscrier, Fifth World Congr. Gynaec Obstet. Butterworths, Australia (1967). 50. Bates, R. W. und H. Corren, Endocrinology $47,166,182$ (1950).
Priv.-Doz. Dr. B. Runnebaum Priv.-Doz. Dr. K. Holzmann 1. Univ.-Frauenklinik 8 München 15

Maistr. 11 